\title{
A survey of the benthic molluscs of the Dampier Archipelago, Western Australia
}

\author{
Shirley M. Slack-Smith and Clay W. Bryce \\ Department of Aquatic Zoology (Molluscs), Western Australian Museum, Francis Street \\ Perth, Western Australia 6000, Australia \\ email: shirley.slacksmith@museum.wa.gov.au \\ clay.bryce@museum.wa.gov.au
}

\begin{abstract}
A total of 695 molluscan species was recorded from intertidal and subtidal surveys conducted at the Dampier Archipelago, Western Australia during October 1998 and August-September 1999. There was a considerable degree of overlap in the molluscan faunas of the eastern and the central and western sectors. However, an east/west gradation in bottom type and exposure is associated with a significant difference in the faunas of the two areas. Of the 523 species recorded from the steeper, higher energy eastern sector, 153 were not recorded from the central and western sectors. Similarly, 180 of the 536 species from the generally more sheltered waters of the central and western sectors were not found further east. However, 199 species were recorded from only a single station during the two survey periods, with 92 of these being found only in the eastern sector and 107 found only in the central and western sectors. Such records contrast with those of the 29 species recorded from 20 or more stations. The greatest diversity of molluscan species was recorded from subtidal habitats characterised mainly or exclusively by living or non-living hard substrata. A slightly lower but comparable diversity was recorded from intertidal and subtidal soft substrata. The molluscan diversity of intertidal hard substrata was approximately one-third that occurring on subtidal hard substrata and on the combined intertidal/subtidal substrata. The diversity of the fauna of these three habitat groupings was much greater than that recorded from the mangals and those recorded in close association with plants and other animal groups. More detailed examination of these habitats than was possible during this survey would undoubtedly lead to an increase in these numbers. The overall diversity of molluscan species recorded for the Dampier Archipelago waters during this survey is greater than those of other Western Australian areas between the Houtman Abrolhos and the western Kimberley areas which have been similarly surveyed.
\end{abstract}

\section{INTRODUCTION}

Marine biological surveys of the marine biota of the Dampier Archipelago were carried out during October 1998 and August-September 1999. The aim was to establish an inventory of the species of selected phyla living in the waters of that archipelago and to relate the occurrence of those species to their habitats.

\section{MATERIALS AND METHODS}

Intertidal stations were surveyed during periods of low tide, while subtidal stations were examined with the aid of SCUBA. General descriptions of these stations, with latitudes and longitudes, are given in the Station Lists section of this volume. More detailed descriptions of those survey stations, as they apply to the areas and habitats surveyed for molluscs, are appended to this paper of the present report (Appendices 1 and 2).

The description of the marine mollusc fauna of the Dampier Archipelago presented here results from records of two expeditions, each involving both diving and intertidal collecting methods. The first survey is titled DA1/98 (October 1998) and the second DA3/99 (August-September 1999). These terms are used to prefix the formal numbering of the 35 stations that were examined during each of these surveys, giving a total of 70 stations, numbered chronologically. It should be noted that another survey, DA2/99 (July 1999) using dredging and grab sampling methods, targeted soft sediment habitats. The results of the mollusc component of that survey is presented in Taylor \& Glover (2004).

The mollusc species present at each station were recorded along with a record of the habitat in which 
each was found. Voucher specimens of each species are retained within the collections of the Western Australian Museum.

Habitats within each station surveyed during the 1998 and 1999 intertidal/dive surveys (DA1/98 and DA3/99) were grouped to reflect their relevance to molluscs, their biology and life history. The area comprising each of the stations surveyed generally encompassed several habitats grading into one another. As an example, a subtidal rocky reef might merge through a zone of broken coral rubble with sandy pockets to an open sandy plain. Many similar combinations are to be found within the waters of the Dampier Archipelago.

An initial attempt was made to estimate the abundance of the mollusc species at individual stations. However, due to the cryptic nature of many species, together with constraints related to conservation of the habitats and other organisms and to the time available for surveying each station, this procedure was discontinued.

\section{Habitats}

The habitat groupings adopted were:

\section{Intertidal hard substrata (IH)}

This is an intertidal zone consisting mainly of coral rubble, rocky reef and/or shoreline rock. These hard substrata may be covered with a thin coating of sediment. Animals recorded from such a substratum may either burrow into, cement or adhere to it, or shelter within crevices. They do not move freely within the sediment.

Subtidal hard substrate (SH)

As above but situated below extreme low water spring tide level.

\section{Soft sediment (S)}

Intertidal and subtidal sand, silt or mud habitats. In such habitats, mollusc species may be infaunal or epifaunal.

\section{Associated with another organism (AP, AA)}

This habitat type applies to those molluscs whose survival strategy is reliant on an intimate and obligatory association with another life form. Occasional, non-obligatory associations of this type are not included here

Associated/Plant (AP)

Molluscs limited to living on sea grasses or algae.

Associated/Animal (AA)

Molluscs limited to living either on or in another living animal (live corals, gorgonians etc.).

\section{Mangals (M)}

In this habitat, mollusc species may occur among or on mangrove trees (including their pneumatophores or other roots), dead logs or other litter and mud. However, during this survey, time constraints did not allow adequate sampling of infaunal species.

\section{RESULTS}

\section{Species Lists}

A tabular list of the 695 mollusc species found during surveys DA1/98 and DA3/99 is given in Appendix 1 . The registration numbers of one or more specimen lots are given for those species for which the identity is currently unknown or uncertain.

For each species listed in Appendix 2 the survey station or stations at which that species was recorded and/or collected is given by the station number, together with an indication of the habitat or habitats in which that species was generally found.

\section{DISCUSSION}

As anticipated from previous studies on the mollusc fauna of the Dampier Archipelago and of areas further to the south, the species recorded during these surveys were all of tropical and subtropical affinity, typical of the fauna of the IndoWest Pacific Region.

\section{Comparative Mollusc Diversity of the Dampier Archipelago Waters}

The total of 695 mollusc species recorded during this Dampier Archipelago Survey is higher than that recorded for any other locality in the north of Western Australia that has been similarly surveyed (Table 1). However, the return per unit of effort (calculated as the number of species recorded per person-day) is lower than that of the 1995 Muiron Islands-Exmouth Gulf survey (Slack-Smith and Bryce, 1996), of the 1996 Western Kimberley survey (Bryce, 1997) and of the 1995 Bernier-Dorre Islands survey (Slack-Smith and Bryce, 1995). When each section (DA1/98 and DA3/99) of the Dampier Archipelago Survey is considered separately, the figures for "species recorded per unit effort" compare more favorably with those of surveys in these other areas. Some caution is needed in interpreting these results, however, as other factors may be involved, such as the amount of collecting time lost due to weather, travel and station depth.

These figures reflect a degree of repetition in the species lists for the stations surveyed and so are indicative of the widespread distribution of many species within the archipelago waters. Such species are, in general, widely distributed within northern Australian waters and even throughout the central Indo-West Pacific Region. 
Of the 523 species of molluscs recorded from the eastern sector of the Dampier Archipelago during the DA1/98 survey, 153 were not recorded from the central and western sectors during the DA3/99 survey. Conversely, of the 536 species recorded during DA3/99, 180 were not found during DA1/98 (Appendix 1).

These results emphasise the difference in the nature of the habitats represented in the steeper, high energy, oceanic eastern zone of the Archipelago as distinct from those of the generally shallower, more sheltered waters of the central and western zones.

One hundred and ninety nine species of the overall total of 695 were recorded from single stations. Of these, 92 were found only during the survey DA1/98 and 107 only during DA3/99. Such records contrast with the number of species recorded from many stations, particularly those from 20 or more, as listed in the results section.

\section{Widespread and Abundant Mollusc Species}

Many molluscs are cryptic and/or nocturnal. Due to logistical constraints, it proved impossible to reliably estimate the abundance of each species at the stations surveyed. However, the data collected (see the number of stations at which each species was recorded in Appendix 1) does reflect those benthic mollusc species most often encountered. These species are not only the most widespread within the surveyed waters but are generally the most abundant. A list of the species that occurred at $>20$ of the 70 survey stations is given below.

\author{
Gastropoda \\ Tectus pyramis (Born, 1778) \\ Angaria delphinus (Linnaeus, 1758) \\ Astralium stellare (Gmelin, 1790) \\ Turbo petholatus Linnaeus, 1758 \\ Cerithium novaehollandiae A. Adams, 1855 \\ Rhinoclavis brettinghami Cernohorsky, 1974 \\ Strombus urceus Linnaeus, 1758 \\ Cypraea eglantina Duclos, 1833 \\ Cronia avellana (Reeve, 1846) \\ Morula margariticola (Broderip, 1832) \\ Morula spinosa (H. and A. Adams, 1853) \\ Thais echinata (Blainville, 1832) \\ Melo amphora (Solander, 1786) \\ Cymbiola oblita (Smith, 1909) \\ Phyllidia coelestis (Bergh, 1869) \\ Phyllidiella pustulosa (Cuvier, 1804)

\section{Bivalvia} \\ Arca ventricosa Lamarck, 1819 \\ Barbatia (Barbatia) amygdalumtostum (Röding, \\ 1798) \\ Septifer bilocularis (Linnaeus, 1758) \\ Pinna bicolor Gmelin, 1791
}

Pinna deltodes Menke, 1843

Pinctada albina (Lamarck, 1819)

Isognomon isognomum (Linnaeus, 1758)

Isognomon legumen (Gmelin, 1791)

Malleus malleus (Linnaeus, 1758)

Complicachlamys wardiana Iredale, 1939

Decatopecten radula (Linnaeus, 1758)

Plicatula australis Lamarck, 1819

Dendostrea folium (Linnaeus, 1758)

\section{Habitats}

A mollusc species may favor one habitat, though it may also occur in neighboring habitats where similar substrates or other essential environmental factors are available. Similarly, the "hosts" of many animal or plant-associated species, as well as the species of symbionts, predators or parasites, may exhibit flexibility in their environmental requirements.

This survey showed that, in the waters of the Dampier Archipelago, subtidal hard substrata support the most diverse mollusc fauna, with 307 of the 695 species recorded from that habitat type. By contrast, mollusc diversity was shown to be least on subtidal reefs supporting a dense growth of living corals. Molluscs inhabiting such aesthetically attractive habitats are of two main types - those that shelter (during the day, at least) amid the "under storey" beneath the living coral growth and those which live in a more intimate association with the live coral itself.

A greater variety of molluscs occur in other habitats, such as reef areas (both intertidal and subtidal) consisting principally of hard rock substrata, dead coral slabs, rubble and sand pockets, with a little live coral and other colonial forms such as sponges and soft corals. Such areas generally support a variety of algal and, more rarely, sea grass communities and so support herbivorous as well as carnivorous species.

About $16 \%$ of the mollusc species found on subtidal hard substrata can also live on intertidal equivalents. However, of the 101 species recorded from intertidal hard substrata, over $50 \%$ were also found subtidally.

Extensive intertidal and subtidal sandy plains often appear almost bereft of molluscs, as well as other biota. However, this habitat generally supports a wide diversity and often high concentrations of sand dwelling species, both infaunal and epifaunal filter feeders, detritivores and carnivores. Some sand-dwelling species may also inhabit small sand pockets within reef flats and subtidal reefs. The 277 mollusc species recorded as soft-substratum dwellers have not been separated into intertidal or subtidal habitats as there is much overlap. Only two soft-substratum dwellers seem to be also associated with hard substrata. Various venerid bivalves apparently benefit from the extra 
protection from predators (fish, birds, etc.) gained from living among rocks and the solecurtid Azorinus ?minutus was found within a mangal.

Twenty one mangal-associated mollusc species were recorded during this survey. They included almost all the gastropods belonging to the Potamididae, Ellobiidae and Onchidiidae, a neritid and two species of littorinid gastropods and the mytilid mussel Stavelia horrida. These all appeared to be restricted to the mangals. Some mangal species, such as the rock oysters and the bivalve Azorinus, were less restricted in their habitat.

Forty three mollusc species were associated with other organisms but little detail is known of the degree and specificity of most of these associations. Some pteriid species of the genera Pteria and Electroma appear to survive best when protected from predators by the hydroids and gorgonians to which they attach, although a very few individuals were found living in rock crevices. Some apparently conspecific individuals of the mytilid genus Lithophaga were found within both dead and living coral skeletons, even though Kleeman (1980) indicated that species on the Great Barrier Reef in Queensland and in Aqaba inhabited only dead or living coral, but not both. The oyster Ostrea tuberculata and the pectinid Hemipecten forbesianus showed little if any selectivity of the living coral species to which they attached.

\section{Diversity}

Discounting the stations DA1/98/05 and DA1/ $98 / 34$, at which molluscs were not surveyed (although specimens were collected by workers on other groups), the 68 stations surveyed for molluscs are divided, for this purpose, into two groups subtidal and intertidal.

It should be noted, however, that these groups might overlap to some extent. Onshore sections of some stations, which were surveyed by diving at high tide, would be exposed at low water springs, if not at low water neaps. Conversely, the offshore sections of some station areas surveyed on foot during low tides were at or below the level of low water neap if not low water spring tides. In addition, some molluscan species inhabit areas both above and below low water levels.

The numbers of molluscan species recorded from the 45 'subtidal' survey stations ranged from 14 to 85 , with an average diversity of 54.80 species/ station. Of these 45 stations, DA1/98/04 and 33, and DA3/99/37, 44, 47, 67 and 70 exhibited the greatest diversity (see Appendix 1). At least 75 species were recorded from each, with an average of 79.7 species/station.

The numbers of molluscan species recorded from the 23 'intertidal' survey stations ranged from 34 to 120 , with an average diversity of 81.04 species/ station. Of these 23 stations, DA1/98/11, 23 and 31, and DA3/99/38 and 54 exhibited the greatest diversity (see Appendix 1), with 101 species or more recorded from each and an average of 108.40 species/station.

\section{REFERENCES}

Bryce, C.W. (1997). Molluscs. In Walker, D.I. (ed.), Marine Biological Survey of the Central Kimberley Coast, Western Australia (National Estates Grant Program Project), December, 1996: 46-57. University of Western Australia, Perth.

Kleemann, K.H. (1980), Boring bivalves and their host corals from the Great Barrier Reef. Journal of Molluscan Studies 46: 13-54.

Slack-Smith, S.M. and Bryce, C.W. (1995). Molluscs. In Hutchins, J.B., Slack-Smith, S.M., Marsh, L.M., Jones, D.S., Bryce, C.W., Hewitt, M.A. and Hill, A. (eds), Marine Biological Survey of Bernier and Dorre Islands. Report to the Ocean Rescue 2000 Program (Project number G009/93), October, 1995: 57-81. Western Australian Museum and Department of Conservation and Land Management, Perth.

Slack-Smith, S.M. and Bryce, C.W. (1996). Molluscs. In Hutchins, J.B., Slack-Smith, S.M., Bryce, C.W., Morrison, S.M. and Hewitt, M.A. (eds), Marine Biological Survey of the Muiron Islands and the eastern shore of Exmouth Gulf. Report to the Ocean Rescue 2000 Program (Project number G0012/94), February, 1996: 64-101. Western Australian Museum, Perth.

Taylor, J.D. and Glover, E.A. (2004). Diversity and distribution of subtidal benthic molluscs from the Dampier Archipelago, Western Australia; results of the 1999 dredge survey (DA2/99). In Jones, D.S. (ed.), Report on the Results of the Western Australian Museum/ Woodside Energy Ltd. Partnership to explore the Marine Biodiversty of the Dampier Archipelago, Western Australia, 1998-2002. Records of the Western Australian Museum Supplement 66: 247-291.

Wells, F.W. and Bryce, C.W. (1995). Molluscs. In Wells, F.E., Hanley, J.R. and Walker, D.I. (eds), Marine Biological Survey of the Southern Kimberley, Western Australia: 101-117. Western Australian Museum, Perth.

Wells, F.E., Slack-Smith, S.M. and Bryce, C.W. (2000). Molluscs. In Berry, P.F. and Wells, F.E. (eds), Survey of the Marine Fauna and Habitats of the Montebello Islands, Western Australia. Records of the Western Australian Museum, Supplement 59: 29-46. 
Table 1 Comparison of results of present (DA1/98, DA3/99) and similar past surveys off the northern coasts of Western Australia (listed from north to south).

\begin{tabular}{|c|c|c|c|c|c|c|}
\hline $\begin{array}{l}\text { Surveyed } \\
\text { Locality }\end{array}$ & Year & $\begin{array}{c}\text { Nos } \\
\text { workers }\end{array}$ & $\begin{array}{l}\text { Survey duration } \\
\text { (days) }\end{array}$ & $\begin{array}{c}\text { Total } \\
\text { species }\end{array}$ & $\begin{array}{l}\text { Species per day } \\
\text { per person }\end{array}$ & Source \\
\hline $\begin{array}{l}\text { Western } \\
\text { Kimberley }\end{array}$ & 1996 & 1 & $\begin{array}{c}13 \\
\text { (13 person days) }\end{array}$ & 292 & 22.46 & Bryce (1997) \\
\hline $\begin{array}{l}\text { Southern } \\
\text { Kimberley }\end{array}$ & 1994 & 2 & $\begin{array}{c}13 \\
\text { (26 person days) }\end{array}$ & 232 & 8.99 & $\begin{array}{l}\text { Wells and Bryce } \\
\text { (1994) }\end{array}$ \\
\hline $\begin{array}{l}\text { Dampier Arch. } \\
\text { (DA1/98, } \\
\text { DA3/99) }\end{array}$ & $\begin{array}{c}1998 \text { and } \\
1999\end{array}$ & 2 & $\begin{array}{c}26 \\
\text { (52 person days) }\end{array}$ & 695 & 13.4 & Present surveys \\
\hline $\begin{array}{l}\text { Dampier Arch. } \\
\text { DA1/98 }\end{array}$ & 1998 & 2 & $\begin{array}{c}13 \\
\text { (26 person days) }\end{array}$ & 523 & 20.1 & Present survey \\
\hline $\begin{array}{l}\text { Dampier Arch. } \\
\text { DA3/99 }\end{array}$ & 1999 & 2 & $\begin{array}{c}13 \\
\text { (26 person days) }\end{array}$ & 536 & 20.6 & Present survey \\
\hline Montebello Islands & 1993 & 3 & $\begin{array}{c}17 \\
\text { (51 person days) }\end{array}$ & 631 & 12.82 & Wells et al. (2000) \\
\hline $\begin{array}{l}\text { Muiron Islands } \\
\text { and east coast } \\
\text { of Exmouth Gulf }\end{array}$ & 1995 & 2 & (24 person days) & 655 & 27.29 & $\begin{array}{l}\text { Slack-Smith and } \\
\text { Bryce (1996) }\end{array}$ \\
\hline $\begin{array}{l}\text { Bernier and Dorre } \\
\text { Islands, Shark Bay }\end{array}$ & 1995 & 2 & $\begin{array}{c}12 \\
\text { (24 person days) }\end{array}$ & 425 & 16.86 & $\begin{array}{l}\text { Slack-Smith and } \\
\text { Bryce } 1995\end{array}$ \\
\hline $\begin{array}{l}\text { Houtman Abrolhos } \\
\text { Islands }\end{array}$ & $\begin{array}{c}\text { To } \\
\text { May } 1994\end{array}$ & Numerous & $\begin{array}{l}\text { Accumulated } \\
\text { over some years }\end{array}$ & 492 & $?$ & $\begin{array}{l}\text { Wells and Bryce } \\
1997\end{array}$ \\
\hline
\end{tabular}

Table 2 Distribution of mollusc species within the waters of the Dampier Archipelago: analysis of the species list.

\begin{tabular}{lr}
\hline \multicolumn{1}{c}{ Species } & Numbers \\
\hline Total number of species recorded from the two intertidal/diving surveys, DA1/98 and DA3/99, each & 695 \\
encompassing 35 survey stations & 523 \\
Total number of species recorded only from survey DA1/98 & 536 \\
Total number of species recorded only from survey DA3/99 & 153 \\
Number of species recorded as limited to survey DA1/98 & 180 \\
Number of species recorded as limited to survey DA3/99 & 199 \\
Total number of species recorded from DA1/98 and DA3/99 combined, which were represented by & 92 \\
only a single record & 107 \\
Number of species recorded only from survey DA1/98, which were represented by only a single record &
\end{tabular}

Table 3 Mollusc diversity within habitat groupings.

\begin{tabular}{lc}
\hline \multicolumn{1}{c}{ Habitat grouping } & Number of species \\
\hline Subtidal hard substrata (SH) & 307 \\
Intertidal hard substrata (IH) & 101 \\
Soft substrata (S) & 277 \\
Mangals (M) & 21 \\
Animal associated (AA) & 43 \\
Plant associated (AP) & 7 \\
\hline
\end{tabular}


Appendix 1 Marine mollusc species recorded from the Dampier Archipelago during diving surveys DA1/98 and DA3/99. Habitat key: $\mathrm{IH}=$ intertidal hard substrate; $\mathrm{SH}=$ subtidal hard substrate; $\mathrm{S}=$ soft sediment; $\mathrm{M}=$ mangrove; $\mathrm{A}=$ associated habitat ( $\mathrm{AA}=$ associated with animal, $\mathrm{AP}=$ associated with plant).

\begin{tabular}{lcc}
\hline Taxa & Station number & Habitat \\
\hline Class POLYPLACOPHORA &
\end{tabular}

Family Ischnochitonidae

Ischnochiton sp. (WAM S 18665)

\section{Family Chitonidae}

Acanthopleura gemmata Blainville, 1825

Acanthopleura spinosa (Bruguière, 1792)

Acanthopleura sp. (WAM S 18663)

$2,7,14,17,20,25,28,59,66$

$2,7,14,17,20,28$

14

IH

IH

$\mathrm{IH}$

\section{Class GASTROPODA}

Subclass EOGASTROPODA

\section{Family Lottiidae}

Patelloida mimula (Iredale, 1924)

Patelloida saccharina (Linnaeus, 1758)

Acmeid sp. (WAM S 18654)

\section{Family Patellidae}

Cellana radiata (Born, 1778)

Patella flexuosa (Quoy and Gaimard, 1834)

\section{Subclass ORTHOGASTROPODA}

\section{Family Haliotidae}

Haliotis asinina Linnaeus, 1758

Haliotis crebrisculpta Sowerby, 1914

Haliotis ovina Gmelin, 1791

Haliotis squamata Reeve, 1846

Haliotis varia Linnaeus, 1758

Family Fissurellidae

Diodora jukesii (Reeve, 1850)

Diodora singaporensis (Reeve, 1850)

Emarginula incisura (Adams, 1853)

Emarginula sp. (WAM S 18792)

Hemitoma excentrica (Tredale, 1929)

Macroschisma munita Iredale, 1940

Montfortula variegata (Adams, 1852)

Montfortula rugosa (Quoy and Gaimard, 1834)

Scutus unguis (Linnaeus, 1758)

Family Trochiidae

Astele sp. (WAM S 27125)

Calthalotia baudini (Fischer, 1878) (WAM S 30223)

Calthalotia mundula (Adams and Angas, 1864)

Calthalotia strigata (Adams, 1853)

Chlorodiloma zeus (Fischer, 1874)

Clanculus atropurpureus (Gould, 1849)

Clanculus ?comarilis Hedley, 1912 (WAM S 30221)

Ethalia sp. (WAM S 27110)

Euchelus sp. (WAM S 18541)

Gibbula ?macgillivrayi (WAM S 18579)

Herpetopoma atrata (Gmelin, 1791)

Herpetopoma rubra (Adams, 1853)

Herpetopoma sp. (WAM S 30229)

Hybochelus cancellatus (Krauss, 1848)

Jujubinus gilberti (Montrouzier, 1878)

Jujubinus polychromus (Adams, 1853)

Microtis rubra (Deshayes, 1843)

Monilea callifera (Lamarck, 1822)
$7,17,28,29,38,48,59$

IH

$2,6,7,17,25,28,29,31 \quad$ IH

$7,17,28,30,59$

$\mathrm{IH}$

6,7

$7,17,27,30,31$

IH

IH

$\begin{array}{ll}19,25,35,60 & \mathrm{SH} \\ 9 & \mathrm{SH} \\ 66 & \mathrm{SH} \\ 7,8,14,16,19,20,21,25,37,38,42,44,45,47,48,51,58,59,61 & \mathrm{SH}, \mathrm{IH} \\ 2,6,8,16,17,19,25,45,53,59 & \mathrm{SH}, \mathrm{IH}\end{array}$

$2,7,11,14,23,25,28,54$

$8,25,44,54,61$

$16,28,30,33,38,54,59,66$

29

$4,6,11,12,17,23$

$7,9,14,59,66$

28,30

59

$20,31,36,45,51,54,57,59,62,65$

$6,48,65$

SH,AA

$\mathrm{IH}, \mathrm{SH}, \mathrm{S}$

$6,7,9,14,16,17,19,59$

$2,7,9,14,16,17,19,22,23,28,30,31,41,47,59,66,67$

IH

IH

$6,7,16,47,48$

$6,7,9,18,42,45,57$

$\mathrm{SH}$

SH

$\mathrm{SH}$

$\mathrm{SH}$

$\mathrm{SH}, \mathrm{IH}$

10

$23,30,47$

6,59

$38,41,42,51,54,57,59,62,68$

$\mathrm{SH}, \mathrm{IH}$

$\mathrm{SH}, \mathrm{IH}$

59

1

51,59

$25,28,47,70$

59,70

$42,59,61$

11,41
$\mathrm{SH}$

$\mathrm{SH}$

$\mathrm{SH}$

$\mathrm{SH}$

SH

$\mathrm{SH}$

S 
Taxa

Station number

Habitat

Monodonta labio (Linnaeus, 1758)

Pseudostomatella maculata (Quoy and

Gaimard, 1834)

Pseudostomatella papyracea (Gmelin, 1791)

Pseudostomatella sp. (WAM S 30227)

Stomatella impertusa (Burrow, 1815)

Stomatia phymotis Helbling, 1779

Talopena vernicosa (Gould, 1861)

Tectus fenestratus (Gmelin, 1791)

Tectus pyramis (Born, 1778)

Tectus schleuteri (Sowerby, 1894)

Trochus hanleyanus Reeve, 1843

Trochus histrio Reeve, 1842

Trochus ?histrio Reeve, 1842

$2,7,10,17,23,59,66$

IH

$\mathrm{SH}$

$6,14,16,19,23,25,37,47,51,59,70 \quad \mathrm{SH}$

$3,8 \quad \mathrm{SH}$

$25,27,37,42,45,47,48,51,59,61,70 \quad \mathrm{SH}$

$13,16 \quad \mathrm{SH}$

37,47

$14,23,25,31,35,38,51,54,59,62,66,68 \quad \mathrm{SH}, \mathrm{IH}$

$1,3,8,10,12,13,14,16,18,19,20,21,22,23,24, \quad \mathrm{SH}, \mathrm{IH}$

$25,29,31,32,35,36,37,42,43,44,45,46,47,49,52,53,56$,

$58,61,66,67,70$

37

$2,6,7,8,14,23,25,28,31,38,42,45,51,54,59,62,66 \quad \mathrm{SH}, \mathrm{IH}$

$3,7,12,18,23,35,36,38,44,49,58,63 \quad \mathrm{SH}$

46

Trochus ?stellatus (Gmelin, 1791) (WAM S 27104) 12,16,19,22,25,28?

?Sub-family Solariellinae (WAM S 30225) $38 \quad 38$

Family Turbinidae

Angaria delphinus (Linnaeus, 1758)

$2,6,9,14,16,17,19,28,31,35,37,38,40,43,46,47,48,51,54$,

$\mathrm{SH}, \mathrm{IH}$

Astralium pileolum (Reeve, 1842)

Astralium rotularia (Lamarck, 1822)

Astralium stellare (Gmelin, 1790)

Liotina crassibassis Smith, 1880

Liotina peronii (Kiener, 1839)

Phasianella solida (Born, 1778)

Turbo argyrostomus Linnaeus, 1758

Turbo brunneus Röding, 1791

Turbo cinereus Born, 1798

Turbo petholatus Linnaeus, 1758

Turbo squamosus Gray, 1847

\section{Family Neritidae}

Nerita albicilla Linnaeus, 1758

Nerita balteata Reeve, 1855

Nerita chamaeleon Linnaeus, 1758

Nerita squamulata Le Guillou, 1841

Nerita undata Linnaeus, 1758

$56,58,59,62,64,67,70$

$6,7,9,11,14,16,17,19,21,23,25,31,35,37,38,44,45,48,51,54,66 \quad \mathrm{SH}$

$45,48,51$

$1,6,9,12,13,16,17,18,19,21,22,28,29,32,35,36,37,42,44, \quad \mathrm{SH}$

$45,46,47,48,50,51,54,56,58,59,61,67,69,70$

6,19

$6,7,16,19,23,28$

$7,9,14,16,25,30,37,45,47,48,51$

$3,7,8,12,13,15,16,18,21,22,25,32,37,44,46,53,58,68$

$4,6,16,17,19,25,35,38,43,45,47,48,51,66,68,70$

$7,10,11,14,17,23,25,28,31,38,42,45,51,54,59,62 \quad$ IH

$1,6,8,13,16,18,22,24,33,37,40,42,43,47,48,56,57,60,6165,68,70 \mathrm{IH}$

$7,10,11,25,31,35,38,48,51,54,62,64$

IH

$7,10,23,38,59$

$11,17,31,54,59,62,66$

$17,20,25 \quad \mathrm{IH}$

2 IH

$10,17,20,28,42,54,59,62,66 \quad$ IH

$37,46,54,59,64 \quad \mathrm{SH}$

Family Littorinidae

Littoraria filosa (Sowerby, 1832)

Littoraria pallescens (Philippi, 1846)

Nodilittorina trochoides (Gray, 1839)

Nodilittorina vidua (Gould, 1859)

$51,54,59,62$

28,31

10,14

7,10

$\mathrm{M}$

IH

$\mathrm{IH}$

Family Rissoidae

cf. Zebina gigantea Deshayes, 1850

64

$?$

Family Turritellidae

Archimediella fastigiata (Adams and Reeve, 1848) 30

30

S

Family Modulidae

Modulus tectum (Gmelin, 1791)

$6,7,16,25,32,35,36,37,44,47,53,54,58,70$

$\mathrm{SH}$

Family Planaxidae

Planaxis sulcatus (Born, 1780)

$7,10,11,14,17,20,25,28,38,45,51,54,59,62,66$

IH

Family Capulidae

Cheilea equestris (Linnaeus, 1758)

$16,17,21,27,31,32,38,46,47,48,57,59$

$\mathrm{SH}$ 
Family Xenophoridae

Xenophora indica (Gmelin, 1791)

Family Siliquariidae

Siliquaria ponderosa (Mörch, 1860)

$4,10,11,13,18,23,31,35,37,38,54,59 \quad \mathrm{SH}$

Siliquaria sp. cf anguina (Linnaeus, 1758)

Family Vermetidae

Serpulorbis sp. A (WAM S 18604)

Beaded vermetid (WAM S 18603)

$1,4,24,33,40,47,53,56,57,60 \quad \mathrm{SH}$

11

Family Cerithiidae

Cerithium balteatum Philippi, 1848

Cerithium coralium Kiener, 1841

Cerithium echinatum Lamarck, 1822

Cerithium novaehollandiae A. Adams, 1855

Cerithium torresi Smith, 1884

Cerithium traillii Sowerby, 1855

Cerithium zonatum (Wood, 1828)

Pseudovertagus aluco (Linnaeus, 1758)

Rhinoclavus bituberculata (Sowerby, 1865)

Rhinoclavis brettinghami Cernohorsky, 1974

Rhinoclavus articulata (Adams and Reeve, 1850)

Rhinoclavus fasciatus (Bruguière, 1792)

Rhinoclavus kochi (Philippi, 1848)

$6,4,19,44,54$

59

$3,4,6,13,19,21,32$

$1,3,4,6,11,12,14,16,17,19,20,21,22,23,24,28,29,31,32,33,35, \quad S$

$36,37,40,41,43,45,47,48,50,51,52,53,54,56,57,58,61,62$,

$65,67,70$

11,62

$19,35,61$

$6,7,10,11,14,19,35,53,54,59,62,66,70$

$7,10,11,14,31,35,38,41,51,54,59$

$9,14,45,51$

$6,7,8,13,14,16,22,25,31,33,37,43,44,46,47,49,50,51,58,62,65,70 \mathrm{~S}$ 38

$7,10,11,38,41,51,54,59,62,66$

41,68

Rhinoclavus vertagus (Linnaeus, 1758)

$2,10,23$

Velacumantus australis (Quoy and Gaimard, 1834) 2,6

$\mathrm{S}$

$\mathrm{S}$

Family Potamidiidae

Cerithidea cingulata (Gmelin, 1791)

Cerithidea reidi Houbrick, 1986

Clypeomorus batillariaeformis Habe and Kosuge, 1966

Clypeomorus bifasciata (Sowerby, 1855)

Telescopium telescopium (Linnaeus, 1758)

Terebralia palustris (Linnaeus, 1767)

Terebralia semistriata Mörch, 1852

$31,54,62$

31

$7,11,14,20,28,54,59,62$

M

59

62

$2,31,54,59,62,66$

$31,54,59$ .

.

Family Truncatellidae

Truncatella sp. (WAM S 22039)

Family Strombidae

Lambis lambis (Linnaeus, 1758)

Strombus campbelli Griffith and Pidgeon, 1834

Strombus labiatus (Röding, 1798)

Strombus mutabilis Swainson, 1921

$2,11,14,17,20,23,30,38,41,42,44,54,56,62$

7

$11,17,38,44,45$

$2,7,9,10,11,17,20,23,25,31,34,35,37,38,42,45,48,51,54, \quad S$ $59,62,66,69$

Strombus vomer (Röding, 1798)

Terebellum terebellum (Linnaeus, 1758)

$9,11,14,17,19,25,37,40,47,70$

41

$S$

Family Vanikoridae

Vanikoro cancellata (Lamarck, 1822) 67

67
31

$\mathrm{SH}$

Vanikoro sigaretiformis (Potiez and Michaud, 1838) 31

Family Calyptraeidae

Crepidula aculeata Gmelin, 1791

$20,26,30,38,39,41,45,51,54$

IH

Family Naticidae

Natica collei (Récluz, 1844)

Natica euzona (Récluz, 1844)

Natica fasciata (Röding, 1791)

41

$7,54,59,66$

20
S

$\mathrm{S}$

$\mathrm{S}$ 
Natica gualteriana (Récluz, 1844)

Natica robillardi Sowerby, 1843

Natica simplex Schepman, 1909

Natica vitellus Linnaeus, 1758

Polinices albumen (Linnaeus, 1758)

Polinices conicus (Lamarck, 1822)

Polinices melanostomus (Gmelin, 1791)

Polinices powisiana (Récluz, 1844)

Polinices simiae (Deshayes, 1838)

Family Cypraeidae

Cypraea carneola Linnaeus, 1758

Cypraea gracilis Gaskoin, 1849

Cypraea lynx Linnaeus, 1758

Cypraea miliaris Gmelin, 1791

Cupraea lutea Gmelin, 1791

Cypraea annulus Linnaeus, 1758

Cypraea chinensis Gmelin, 1791

Cypraea clandestina Linnaeus, 1767

Cypraea cribraria Linnaeus, 1758

Cypraea cylindrica Born, 1778

Cypraea eglantina Duclos, 1833

Cypraea erosa Linnaeus, 1758

Cypraea errones Linnaeus, 1758

Cypraea flaveola Linnaeus, 1758

Cypraea helvola Linnaeus, 1758

Cypraea hirundo Linnaeus, 1758

Cypraea isabella Linnaeus, 1758

Cypraea limacina Lamarck, 1810

Cypraea pallidula Gaskoin, 1849

Cypraea quadrimaculata Gray, 1824

Cypraea staphylaea Linnaeus, 1758

Cypraea subviridus Reeve, 1835

Cypraea talpa Linnaeus, 1758

Cypraea alisonae Burgess, 1983/teres Gmelin, 1791

Cypraea ursellus Gmelin, 1791

Cypraea tigris Linnaeus, 1758

Cypraea vitellus Linnaeus, 1758

Cypraea caurica Linnaeus, 1758

Cypraea caputserpentus Linnaeus, 1758

Cypraea moneta Linnaeus, 1758

Cypraea hammondae Iredale, 1939

Cypraea cicercula Linnaeus, 1758

Family Ovulidae

Prosimnia semperi (Weinkauff, 1881)

Phenacovolva sp. 1 (WAM S 27000)

Phenacovolva sp. 2 (WAM S 27002)

Family Triviidae

Trivia oryza (Lamarck, 1810)

Family Epitoniidae

Epitonium sp. (WAMS 27048)

Epitonium costulatum Kiener, 1839

Family Eulimidae

Apicalia sp. cf. brazieri (Angas, 1877)

Eulimid sp. (WAM S 27010)

Family Ficidae

Ficus sp. cf. subintermedia (Orbigny,1852)

Ficus eospilla (Péron, 1807)

$\begin{array}{ll}11,38,45,48,54,59,66,70 & \mathrm{~S} \\ 38,62 & \mathrm{~S} \\ 54,56,66 & \mathrm{~S} \\ 45 & \mathrm{~S} \\ 38 & \mathrm{~S} \\ 11,38,62,59 & \mathrm{~S} \\ 7,37,38,47 & \mathrm{~S} \\ 51,68,70 & \mathrm{~S} \\ 65 & \mathrm{~S}\end{array}$

40

SH

$36,45,47,48,54,56,66,70 \quad$ IH

$45,48,51 \quad \mathrm{SH}$

$37,68,69 \quad$ SH

$54 \quad$ SH

$48,66 \quad \mathrm{IH}$

$3,70 \quad \mathrm{SH}$

$7,8,16,21,36,37,38,45,46,51 \quad$ SHI

$8,12,15,44,57,60 \quad$ SH

$4,8,12,17,18,19,20,21,23,24,25,28,31,32,35,36,45,46,64,70 \quad$ SH

$3,6,8,12,14,15,16,18,19,20,21,22,25,26,36,37 \quad \mathrm{SH}$

$40,44,45,46,47,49,52,53,57,62,69$

$3,10,12,13,14,15,18,19,21,24,32,36,44,53,58,60,64 \quad \mathrm{SH}$

$11,14,28,38,45,48,51,54,59,62,66 \quad$ IH

$42 \mathrm{SH}$

$16,21,22,32,37,46,47,50 \quad \mathrm{SH}$

$3,4,6,8,21,28,45 \quad \mathrm{SH}$

$7,13,18,44 \quad$ SH

$21,40,46 \quad$ SH

$32 \mathrm{SH}$

12 SH

$3,13,16,38,45 \quad$ SHI

$14,33,37,41,42,44,48,51,59,70 \quad$ SH

$21 \quad \mathrm{SH}$

$12,21,40 \quad \mathrm{SH}$

$3,4 \quad \mathrm{SH}$

$18,39 \quad$ SH

$1,17,18,24,38,44,45,48,51,53,54 \quad \mathrm{SH}$

$1,4,6,9,13,16,20,33,12,36,40,45,47,48,54,62 \quad \mathrm{SH}$

$3,8,10,15,26,42,46,48,58 \quad$ SH

$7,8,11,38,48,51,59,66 \quad$ IH

$22,47 \quad \mathrm{SH}$

$67-\mathrm{SH}$

8

41,68 AA

39 AA

$3,6,10,12,21,22,31,32,64,47,48,54 \quad \mathrm{SH}$

49 AA

53 AA

67 AA

$48-$ AA

445

$41,55 \quad S$ 
Family Velutinidae

Chelynotus tonganus Quoy and Gaimard, 1832

21,49

$\mathrm{SH}$

Family Ranellidae

Cymatium labiosum (Wood, 1828)

Cymatium pileare (Linnaeus, 1758)

Cymatium vespaceum (Lamarck, 1822)

Cymatium sarcostomum (Reeve, 1844)

Gyrineum lacunatum (Mighels, 1845)

\section{Family Bursidae}

Bursa granularis (Röding, 1798)

Family Cassidae

Phalium bandatum (Perry, 1811)

Semicassis pyrum (Lamarck, 1822)

Semicassis bisulcata (Schubert and Wagner, 1829)

$7,45,54$

$\mathrm{SH}$

$21,24,36,37$

$4,9,11,12,17,23,47,48,51,54,56,57,62,64,66$

57

51

\section{$\mathrm{SH}$}

$\mathrm{SH}$

$\mathrm{SH}$

$\mathrm{SH}$

$4,25,32,39,40,47$

$\mathrm{SH}$

$7,46,56,57,65,68,69$

25,69

69

$S$

$S$

$S$

Family Tonnidae

Tonna allium (Dillwyn, 1817)

Tonna tessellata (Lamarck, 1816)

23,25

69

$S$

S

\section{$23,41,57,59$}

IH

$3,4,21,22,32,40,43,46,49,50,52,69,70$

30

30,57

$23,33,22$

11,23

$11,41,68$

$6,20,37,45,47,48,51,55,56,64,67,70$

6

48

$11,41,57,69$

13

56

70

4,40

27 AA

$31 \quad$ AA

$2,3,4,5,6,7,8,9,10,11,12,13,14,16,17,18,19,2021,22,23,24, \quad$ SH

$25,26,27,28,29,30,31,32,33,36,37,40,42,43,44,45,46,47,48$,

$50,51,53,54,56,57,58,59,61,62,64,65,66,67,70$

$1,7,36,38,44,48,51,54,58,60,63$

$1,2,6,7,8,10,11,12,13,14,17,19,20,21,24,25,28,29,31,32$

$36,38,42,44,45,48,51,54,58,59,60,62,63,66,68$

$1,8,12,16,18,19,21,24,25,29,33,58,60,61$

$7,10,11,25,28,45,48,59,62$

$3,4,8,12,13,15,17,18,19,20,21,22,24,26,27,29,32,35,36, \quad \mathrm{SH}$

$37,39,43,44,45,46,50,56,57,58,60,63,67$

$7,10,25,28,46$

$5,8,46$

Thais alouina Röding, 1798

Thais echinata (Blainville, 1832)

Drupella cornis (Röding, 1798)

Pinaxia versicolor (Gray, 1839)

$43,44,45,46,48,49,50,51,52,53,54,56,57,58,60,63,65,67,69,70$

$8,18,22,29,32,36,58,60,63$

$9,37,70$

$\mathrm{SH}$

$\mathrm{SH}$

$\mathrm{SH}$

$\mathrm{IH}$

$\mathrm{SH}$

IH

$\mathrm{SH}$

AA

AA?

$43,45,48,51$

$\mathrm{SH}$

$6,14,17,19,20,45,48,65$

IH

$6,9,31,34,37,45,47,48,51$

AP

47

Mitrella albina (Kiener, 1841)
AP 


\section{Family Buccinidae}

Cantharus fumosus (Dillwyn, 1817)

Cantharus erythrostomus (Reeve, 1846)

Cominella acutinodosa (Reeve, 1846)

Pisanea ignea (Gmelin, 1791)

Phos sculptilis Watson, 1886

Family Colubrariidae

Colubraria sp. (WAM S 22036)

Family Turbinellidae

Tudivasum inermis (Angas, 1878)

Family Melongenidae

Syrinx artıanus (Linnaeus, 1758)

Family Nassariidae

Nassarius clarus (Marrat, 1877)

Nassarius dorsatus (Röding, 1798)

Nassarius glans (Linnaeus, 1758)

Nassarius pauperus (Gould, 1850)

Nassarizis conoidalis (Deshayes, 1832)

Nassarius albinus (Thiele, 1930)

Nassarius albescens (Dunker, 1846)

Hebra horrida (Dunker, 1847)

\section{Family Fasciolariidae}

Latirus turritus (Gmelin, 1791)

Peristernia incarnata (Kiener, 1840)

Latirus walkeri Melvill, 1895

Latirus paetelianus (Kobelt, 1876)

Fusinus colus (Linnaeus, 1758)

Family Harpidae

Harpa amouretta Röding, 1798

$38,54,59,62,66$

$2,10,11,54,62$

$6,8,17,31,51,55,57,61,68$

$38,47,62$

38

62

38,54

38

$1,8,12,14,15,18,19,22,25,29,37,44,45,46,48,51,58,63,69,70$

$\mathrm{SH}$ $3,37,44,45,48,51,53,58,70$

\section{$17,45,51,56,61$}

$1,6,17,25,33,51,69,70$

56

$\mathrm{SH}$

IH

$\mathrm{SH}$

$\mathrm{SH}$

\section{Family Olividae}

Ancillista muscae (Pilsbry, 1926)

Ancillista cingulata (Sowerby, 1830)

Oliva caldania Duclos, 1835

\section{Family Mitridae}

Mitra scutulata (Gmelin, 1791)

Mitra fraga Quoy and Gaimard, 1833

Pterygia sinensis (Reeve, 1844)

Pterygia crenulata (Gmelin, 1791)

"Ziba" flammea (Quoy and Gaimard, 1833)

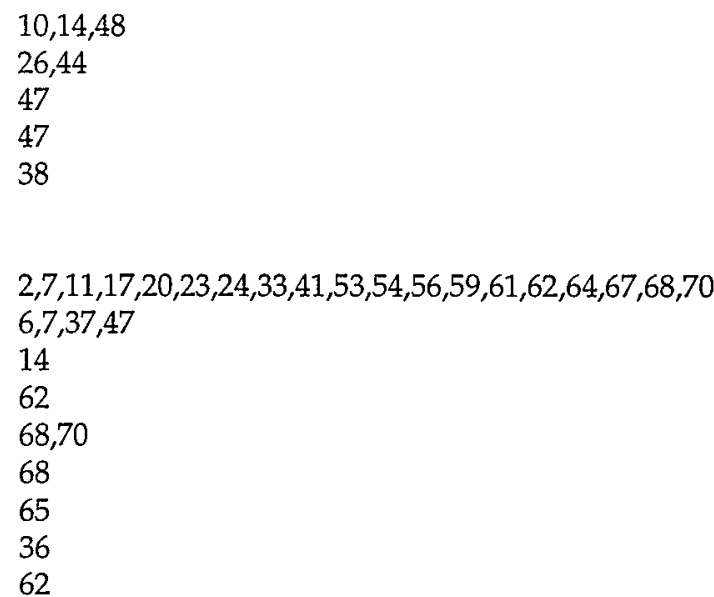

$\mathrm{SH}$

Family Costellariidae

Vexillum vulpeculum (Linnaeus, 1758)

Vexillum pacificum (Reeve, 1845)

Vexillum microzonias (Lamarck, 1811)

Vexillum suluense (Adams and Reeve, 1850)

Vexillum mirabile (A. Adams, 1853)

Vexillum amanda (Reeve, 1845)

Vexillum unifasciatum (Wood, 1828)

Vexillum aureolineatum Turner, 1988

Vexillum radix (Sowerby, 1874 ) 
Family Volutidae

Melo amphora (Solander, 1786)

Cymbiola oblita (Smith, 1909)

Amoria grayi (Ludbrook, 1953)

Amoria jamrachi Gray, 1864

Amoria ellioti (Sowerby, 1864)

Amoria praetexta (Reeve, 1849)

\section{Family Conidae}

Conus geographus Linnaeus, 1758

Conus glans Hwass in Bruguière, 1792

Conus miliaris Linnaeus, 1758

Conus monachus Linnaeus, 1758

Conus musicus Hwass in Bruguière, 1792

Conus reductaspiralis Walls, 1979

Conus textile Linnaeus, 1758

Conus vexillum Gmelin, 1791

Conus victoriae Reeve, 1843

Conus trigonus Reeve, 1848

Conus novaehollandiae Adams, 1853

Conus spectrum Linnaeus, 1758

Conus suturatus Reeve, 1844

Conus lividus Hwass in Bruguière, 1792

Conus dorreensis Péron, 1807

\section{Family Turridae}

21,50

$\mathrm{SH}$

3,8,18,21,22,29,40,44,46,58 SH

10

$19,45,51$

13

$13,38,50,56,65,69,70$

$16,21,22,44,45,47,56,65,70$

$12,40,43$

$4,7,9,10,19,23,25,38,41,45,47,48,51,70$

$20,23,25,38,45$

$1,38,47,48,51,69$

$6,16,23,25,51,56,68$

47

43

45,48

$\mathrm{S}$

$\mathrm{SH}$

$\mathrm{SH}$

$\mathrm{S}$

$\mathrm{SH}$

$\mathrm{S}$

$S$

$\mathrm{SH}$

$\mathrm{SH}$

$\mathrm{S}$

$\mathrm{S}$

$S$

AP
Turris crispa (Lamarck, 1816)

Clavus unizonalis (Lamarck, 1822)

Eucithara sp. (WAM S 27039)

Turricula granobalteus (Hedley, 1922)

Turrid sp. (WAM S 27036)

Family Terebridae

Terebra affinis Gray, 1834

Terebra marrowae Bratcher and

Cernohorsky, 1982

Hastula rufopunctata (Smith, 1877)

Family Pyramidellidae

Pyramidella sp. (WAM S 12014)

Pyramidella dolabrata (Linnaeus, 1758)

Pyramidella acus (Gmelin, 1791)

Pyramidella sulcata (A. Adams, 1854)

Colsyrnola sericea Iredale, 1929

Family Architectonicidae

Heliacus variegatus (Gmelin, 1791)

48

$\begin{array}{ll}41 & \mathrm{~S} \\ 48 & \mathrm{~S} \\ 54 & \mathrm{~S} \\ 41 & \mathrm{~S} \\ 41 & \mathrm{~S}\end{array}$

7

12,56

$\mathrm{S}$

14

S

6

68

68

68

47

$\mathrm{S}$

\section{Subclass OPISTHOBRANCHIA}

Family Acteonidae

Pupa sulcata (Gmelin, 1791)

$10,38,41,51,59,62$

38

38

Atys semistriata Pease, 1860

Haminoea cymbalum (Quoy and Gaimard, 1835)

38

$2,7,9,10,11,14,19,23,25,38,45,51,54,58,64,66,70$

S

7

$S$
$S$
$S$
$S$
$S$

$S$
$S$
$S$

$S$
$S$
$S$
$S$
$S$
$S$

Family Bullidae

Bulla ampulla Linnaeus, 1758

Family Hydatinidae

Micromelo undatus (Bruguière, 1792) 
Family Aglajidae

Chelidonura amoena Bergh, 1905

Family Aplysiidae

Aplysia parvula Guilding in Mörch, 1863

Aplysia dactylamela Rang, 1828

$16-\mathrm{SH}$

Dolabella auricularia (Lightfoot, 1786)

Aplysia sp. cf. extrordinaria Allan, 1932

Dolaberifera dolaberifera Cuvier, 1817

$\mathrm{SH}$

Family Elysiidae

Elysia sp. (WAM S 12395)

Thuridilla sp. (WAM S 1462)

Elysia ornata (Swainson, 1840)

Elysiella pusilla Bergh, 1872

Family Gastropteriidae

Sagaminopteron psychedelicum Carlson and Hoff, 1974

\section{Family Pleurobranchiidae}

Pleurobranchus forskalii (Rüppell and

Leuckart, 1828)

Pleurobranchus martensi (Pilsbry, 1896)

Pleurobranchus peroni (Cuvier, 1804)

Family Gymnodoridae

Gymnodoris sp. (WAM S 1468)

Gymnodoris rubropapulosa Brunckhorst, 1993

Gymnodoris sp. cf. citrina (WAM S 12013,

S 12018, S 12030, S 12035)

\section{Family Hexabranchiidae}

Hexabranchus sanguineus (Rüppell and

Leuckart, 1829)

Family Polyceriidae

Nembrotha purpureolineata O'Donoghue, 1924

Nembrotha kubaryana Bergh, 1877

Nembrotha lineolata Bergh, 1905

Tambja gracilis Bergh, 1877

\section{Family Dorididae}

Aphelodoris sp.(WAM S 1469, S 12043)

Discodoris cf. boholiensis Bergh, 1877

Discodoris lilacina (Gould, 1852)

Jorunna funebris (Kelaart, 1858)

Platydoris scabra (Cuvier, 1804)

cf. Jorunna sp. (WAM S 12057)

Asteronotus cespitosus (Hasselt, 1824)

Halgerda brycei Fahey and Gosliner, 2001

$\begin{array}{ll}4,26,40,44,45,50,53 & \mathrm{SH} \\ 4,12,20,50 & \mathrm{SH} \\ 1,7,11,45,51 & \mathrm{IH} \\ 4,17,24 & \mathrm{SH} \\ 31 & \mathrm{IH} \\ 31 & \mathrm{SH} \\ 6,19,20,28,45,54,66 & \mathrm{IH} \\ 60,67 & \mathrm{SH} \\ 3 & \mathrm{AA} \\ 32 & \mathrm{SH} \\ 53 & \mathrm{AP} \\ 40 & \mathrm{SH} \\ 58 & \mathrm{AA} \\ 33 & \mathrm{AA}\end{array}$

Thordissa villosus (Alder and Hancock, 1864)

Trippa ossesa (Kelaart, 1859)

Red dorid sp. (WAM S 12409)

Discodorid sp. (WAM S 12399)

Dorid sp. in Callyspongia (WAM S 12412)

Dorid sp. (WAM S 12056)

Family Chromodorididae 
Chromodoris sp. (WAM S 12617)

Risbecia sp. cf. tyroni (Garrett, 1873)

Glossodoris atromarginata (Cuvier, 1804)

Chromodoris cf. africana Eliot, 1904

Chromodoris coei (Risbec, 1956)

Chromodoris cf. magnifica (Quoy and

Gaimard, 1832)

Chromodoris striatella Bergh, 1877

Chromodoris lineolata (van Hasselt, 1824)

Glossodoris rufomarginata (Bergh, 1890)

Chromodoris verrieri (Crosse, 1875)

Mexichromus mariei (Crosse, 1872)

Hypselodoris whitei (Adam and Reeve, 1850)

Chromodoris colemani Rudman, 1982

Chromodoris tinctoria (Rüppell and

Leuckart, 1828)

Chromodoris cf. tinctoria complex (Rüppell and Leuckart, 1828)

Chromodoris kuniei Pruvot-Fol, 1930

Risbecia sp. (WAM S 12396)

Glossodoris cincta (Bergh, 1888)

Chromodoris fidelis (Kelaart, 1858)

Ceratosoma trilobatum (J.E. Gray, 1827)

Ceratosoma tenue Abraham, 1876

Ceratosoma magnifica (Eliot, 1910)

Family Dendrodorididae

Dendrodoris albobrunnea Allan, 1933

Dendrodoris dennisoni (Angas, 1864)

Family Phyllidiidae

Phyllidia coelestis (Bergh, 1869)

Phyllidia elegans Bergh, 1869

Phyllidia varicosa Lamarck, 1801

Phyllidiella pustulosa (Cuvier, 1804)

Phyllidia ocellata Cuvier, 1804

Phyllidia exquisita Brunckhorst, 1993

Phyllidia babai Brunckhorst, 1993

Family Glaucidae

Moridilla sp. (WAM S 12031)

Ptaeolidea ianthina (Angas, 1864)

Phyllodesmium crypticum Rudman, 1981

Phyllodesmium poindimiei (Risbec, 1828)

Moridilla brockii Bergh, 1888

\section{Family Flabelliniidae}

Flabellina exoptata Gosliner and Willan, 1991

Flabellina rubrolineata (O'Donoghue, 1929)

Cuthona sibogae (Bergh, 1905)

\section{Family Dotidae}

Lomanotus sp. (WAM S 12400)

\section{Family Bornellidae}

Bornella anguilla Johnson, 1893

Bornella stellifera (Adams and Reeve in

Adams, 1848)

\section{SUBCLASS PULMONATA}

\section{Family Siphonariidae}

Siphonaria zelandica Quoy and Gaimard, 1833
37

$\mathrm{SH}$

$1,33,37,58$

$1,3,12,19,27,37,44,56,60$

$3,8,15$

$4,15,21,26,67$

4

48

$6,31,42$

19,22

16

33,55

$16,18,32$

4,15

61,64

37,61

50

37

46,58

40

45,51

48,51

45

$4,22,49$

$\mathrm{SH}$

45,54

$\mathrm{IH}$

$1,3,4,8,13,15,18,20,21,22,24,2732,36,43,44,50,52,56,57,60,63$ $3,4,8,46,67$

$13,15,18,33,36,52,56,58,60$

$1,3,4,8,13,15,18,21,22,24,27,29,32,33,36,39,44,50,52$

$53,56,57,58,60,63,64,67,69$

$1,3,4,13,18,27,33,36,43,49,50,52,53,57,60,64,65,67$

$3,13,50$

69

$\mathrm{SH}$

$\mathrm{SH}$

$\mathrm{SH}$

$\mathrm{SH}$

$\mathrm{SH}$

$\mathrm{SH}$

$\mathrm{SH}$

17

$3,6,17,21,33.43,55$

$\mathrm{SH}$

31

$4,6,16$

48

AA

AA

$\mathrm{SH}$

$4,15,33,40,65,67 \quad \mathrm{SH}$

$15,26,27 \quad \mathrm{SH}$

$26,27,33$. SH

41

AA

8,43

$22,25,37$

$\mathrm{SH}$

$\mathrm{SH}$ 
Family Ellobiidae

Cassidula cf. nucleus (Gmelin, 1791)

10

M

(WAM S 30245)

Melampus sp. (WAM S 30246)

10,28

M

Family Onchidiidae

Onchidium sp. A (WAM S 13911)

Onchidium sp. D (WAM S 13912)

$2,7,14,17,25,54$

31

M

$\mathrm{M}$

\section{CLASS BIVALVIA}

\section{SUBCLASS PROTOBRANCHIA}

Family Nuculidae

Nucula ?superba Hedley, 1902 (WAM S 18446)

$17,30,55,61,62,67,69$

\section{Subclass PTERIOMORPHIA}

\section{Family Arcidae}

Anadara (Anadara) antiquata (Linnaeus, 1758)

Anadara (Cunearca) rotundicostata (Reeve, 1843)

Anadara (Scapharca) ?rufescens (Reeve, 1844) (WAM S 18388)

Anadara (Tegillarca) granosa (Linnaeus, 1758)

Arca ventricosa Lamarck, 1819

Barbatia (Acar) plicata (Dillwyn, 1817)

Barbatia (Acar) sp. (WAM S 18348)

Barbatia (Barbatia) amygdalumtostum (Röding, 1798)

Barbatia (Barbatia) ?coma (Reeve, 1844) (WAM S 18365)

Barbatia (Barbatia) ?helblingii Bruguière, 1789 (WAM S 18405)

Barbatia (Barbatia) foliata (Forskål, 1775)

Barbatia (Barbatia) ?obliquata (Wood, 1828) (WAM S 18398)

Barbatia (Barbatia) ?parvivillosa (Iredale, 1939) (WAM S 18367)

Barbatia (Barbatia) sp. 1 (WAM S 18371)

Barbatia (Barbatia) sp. 2 (WAM S 30230)

Barbatia (Calloarca) tenella (Reeve, 1843)

Trisidos semitorta (Lamarck, 1819)

Family Noetiidae

Arcopsis afra (Gmelin, 1791)

Striarca sp. (WAM S 18401)

Family Glycymerididae

Glycymeris dampierensis Matsukuma, 1984

Tucetona ?angusticosta Lamprell and

Whitehead, 1990 (WAM S 30231)

Tucetona auriflua (Reeve, 1843)

Tucetona odhneri Iredale, 1939

Tucetona sp. (WAM S 30232)

Family Mytilidae

Botula silicula (Lamarck, 1819)

Brachidontes sp. (WAM S 18172)

Gregariella ?otteri (Iredale, 1939) (WAM S 30265)

Lithophaga ?lessepsiana (Vaillant, 1865)

(WAM S 27131)
$7,14,20,23,25,35,38,54,70$

30

$8,10,12,20,30,56,57,69$

$S$

$S$

2 (long dead) $\mathrm{S}$

$1,2,3,4,6,7,8,10,12,17,18,20,21,22,23,24,25,26,28,29,30,32,33$, SH $35,36,37,39,40,44,46,49,50,53,54,56,57,58,59,60,63$,

$64,65,67,68,70$

$23,35,37,48,49,55,56,67,69 \quad \mathrm{SH}$

32

$3,4,8,9,11,12,13,14,15,16,17,18,19,20,21,22,23,24,25,28, \quad \mathrm{SH}, \mathrm{IH}$ $29,31,33,35,36,37,44,47,49,52,54,58,60,63,66,70$

$2,10,11,23,59,62$

$\mathrm{SH}$

$14,8,10,11,24,32,47,49,50,61,64,67,70$

$\mathrm{SH}, \mathrm{IH}$

$9,11,12,15,17,20,23,28,30,31,33,53,54,55,61,63,69$

$\mathrm{SH}, \mathrm{IH}$

$\mathrm{SH}, \mathrm{IH}$

14

$\mathrm{SH}, \mathrm{IH}$

$12,23,33,37,46,49,54$

$\mathrm{SH}, \mathrm{IH}$

$2,10,23,30,35,51$

$\mathrm{SH}, \mathrm{IH}$

$\mathrm{SH}, \mathrm{IH}$

$11,30,41,61$

$S$

$2,10,17,23,28,30,34,35,37,38,45,47,51,59$

2

$\mathrm{SH}, \mathrm{IH}$

$\mathrm{SH}$

$12,14,25,37,38,41,44,45,55,56,57,61,63,65$,

$S$

$67,68,69, W$ coast of East Lewis I.

$56,65,67$

S

$3,46,47,57,65,70$

$20,23,28,30,31,37,38,45,55,61,67$

42,65

$S$

$S$

$31,42,44$

$\mathrm{AA}, \mathrm{SH}$

$6,7,10,17,28,31,38,39,51,59,66, \mathrm{~W}$ coast of East Lewis I. SH,IH

31,49

$\mathrm{SH}$

$\mathrm{AA}, \mathrm{SH}, \mathrm{IH}$ 
Lithophaga ?malaccana (Reeve, 1858) $1,44,51,67$

$\mathrm{AA}, \mathrm{SH}, \mathrm{IH}$

(WAM S 27128)

Lithophaga ?obesa (Philippi, 1847) (WAM S 30234) 44

Lithophaga teres (Philippi, 1846)

Lithophaga sp. (WAM S 30235)

Modiolus albicostatus Lamarck, 1819

$1,4,20,31,33,34,37,42,48,63,70$

67

11,20

Modiolus ?auriculatus Krauss, 1848

$4,6,25,31,38,48,59,62,67$

$\mathrm{AA}$

(WAM S 18168)

Modiolus ?micropterus Deshayes, 1836 (WAM S 30233)

Modiolus ?philippinarum Hanley, 1843

(WAM S 18179)

Modiolus pulvillus Iredale, 1939

Musculus cumingianus Reeve, 1857

Musculus impactus Hermann, 1782

Septifer bilocularis (Linnaeus, 1758)

Stavelia horrida Dunker, 1856

54

$6,7,9,13,16,17,23,28,37,47,49,54,55,61,66,70$

$\mathrm{AA}, \mathrm{SH}, \mathrm{IH}$

$\mathrm{AA}, \mathrm{SH}, \mathrm{IH}$

$\mathrm{SH}$

$\mathrm{SH}$

SH

$\mathrm{SH}$

54

$S$

44,55

18,67

$\mathrm{AA}, \mathrm{SH}$

$1,2,3,4,6,8,10,12,13,14,15,16,17,19,20,21,22,23,24,25$,

$26,27,28,29,30,32,33,34,35,36,38,39,40,42,43,44,46,47$, $48,49,50,51,52,57,58,59,60,61,62,63,64,66,67,69$

$1,14,23,33,45,48,55$

$\mathrm{AA}, \mathrm{SH}$,

$\mathrm{SH}, \mathrm{IH}$

M

Family Pinnidae

Atrina ?pectinata (Linnaeus, 1758) (WAM S 30236) 30,42

Atrina vexillum (Born, 1778)

Atrina sp. (WAM S 18006)

$1,4,25,32,45,53,58$

$20,30,31$

$2,3,4,7,8,11,16,17,23,27,30,31,38,41,42,45,51,53,54$,

$56,57,59,62,64,65,69$

$4,9,11,12,15,16,17,18,21,26,27,31,32,39,40,43,44$,

$47,48,49,51,52,53,54,64,67$

8,32

$\mathrm{SH}$

Family Pteriidae

Electroma alacorvi (Dillwyn, 1817)

Electroma physoides (Lamarck, 1819)

Electroma spadicea (Dunker, 1852)

Pinctada albina (Lamarck, 1819)

$3,7,17,20,22,24,25,29,31$

41,68

$17,21,58.64,65$

$2,4,7,8,9,11,12,14,16,17,19,20,23,24,25,28,29$,

$31,33,35,37,42,43,54,62,63$

Pinctada ?maculata (Gould, 1850) (WAM S 18466) 7,47

Pinctada margaritifera (Linnaeus, 1758)

7,47

$1,3,13,15,18,21,24,26,27,29,32,33,35,40,47$

$23,24,32,36,39,40,44,47,49,51,53,55,57,59,60,61,65$

$15,26,27,52,56,65$

$15,27,33,52,56,69$

\section{$\mathrm{S}$}

$\mathrm{SH}$

$\mathrm{S}$

S

$\mathrm{S}$

Pteria lata (Gray, 1845)

Pteria penguin (Röding, 1798)

\section{9}

$2,23,29, ? 33,54,66$

$1,3,4,8,10,11,12,13,15,16,18,19,20,22,23,24,25,28,29,31,33,35$ ， SH,IH $38,39,40,42,43,44,46,49,50,51,52,53,57,58,59,60,63,64,67,69$ $1,2,3,4,6,7,8,10,13,14,15,17,18,20,25,34,39,40,44,45,46,48$, $49,50,51,52,53,57,58,59,60,63,64,65,66,67,70$

$6,17,26,61,64$

23,30

$1,4,7,8,10,11,13,14,15,18,19,20,22,24,26,29,30,31,32,33,34$ $32,44,45,49,51,54,55,59,60,62,63$

$20,52,54$

55,56

$22,30,55,56,65,67,68,69$

$4,8,10,12,13,16,17,18,21,22,23,27,32,33$,

$36,41,44,45,46,47,53,55,57,61,65,67,70$

$1,4,17,20,36,37,40,44,58,61$
$\mathrm{SH}, \mathrm{IH}$

$\mathrm{SH}, \mathrm{IH}$

$\mathrm{SH}$

$\mathrm{SH}$

$\mathrm{AA}, \mathrm{SH}$

$\mathrm{AA}, \mathrm{SH}$

AA,

$\mathrm{SH}$

$\mathrm{SH}$

$S$

$\mathrm{AA}, \mathrm{SH}$

$\mathrm{AA}, \mathrm{SH}$

AA,SH

IH

$\mathrm{S}$

$\mathrm{SH}, \mathrm{IH}$

$\mathrm{AA}, \mathrm{SH}$ 
Decatopecten radula (Linnaeus, 1758)

Decatopecten strangei (Reeve, 1852)

Excellichlamys spectabilis (Reeve, 1853)

Gloripallium pallium (Linnaeus, 1758)

Hemipecten forbesianus Adams and Reeve, 1849

Laevichlamys squamosa (Gmelin, 1791)

Mimachlamys ?australis (Sowerby, 1842)

Mimachlamys funebris (Reeve, 1853)

Mimachlamys gloriosa (Reeve, 1852)

Mimachlamys lentiginosa (Reeve, 1853)

Mimachlamys scabricostata (Sowerby, 1915)

Scaeochlamys sp. (Lamarck, 1819)

Pectinid sp. 1 (WAM S 18128)

Pectinid sp. 2 (WAM S 12424)

Pectinid sp. 3 (WAM S 12423)

Pectinid sp. 4 (WAM S 18146)

Family Spondylidae

Spondylus albibarbatus Reeve, 1856

Spondylus asperrimus Sowerby, 1847

Spondylus eastae Lamprell, 1992

Spondylus echinatus Schreibers, 1793

Spondylus heidkeae Lamprell and Healy, 2001

Spondylus ocellatus Reeve, 1856

Spondylus spinosus Schreibers, 1793

Spondylus victoriae Sowerby, 1843

Family Plicatulidae

Plicatula australis Lamarck, 1819

P. chinensis Mörch, 1853

P. ?muricata Sowerby, 1873 (WAM S 18806)

Family Anomiidae

Anomia sp. (WAM S 18057)

Monia ?deliciosa Iredale, 1936 (WAM S 18056)

Patro australis (Gray, 1847)

Family Placunidae

Placuna lobata Sowerby, 1871

Family Ostreidae

Alectryonella plicatula (Gmelin, 1791)

Dendostrea folium (Linnaeus, 1758)

Ostrea tuberculata (Lamarck, 1819)

Ostrea sp. (WAM S 30247)

Saccostrea ?commercialis (Iredale and Roughley, 1933) (WAM S 30248)

Saccostrea cucullata (Born, 1778)

Saccostrea echinata (Quoy and Gainard, 1832)

Family Gryphaeidae

Hyotissa hyotis (Linnaeus, 1758)

Hyotissa numisma (Lamarck, 1819)

Hyotissa ?numisma (Lamarck, 1819) (WAM S 30237)

Hyotissa sp. (WAM S 30238)

Family Limidae

Ctenoides annulata (Lamarck, 1819)
$1,8,12,13,14,16,18,20,21,24,25,29,31,33,35$

$36,37,44,45,49,53,58,60,61,64,67$

$30,37,55,56,57 \quad \mathrm{SH}$

$1,3,4,12,13,15,18,21,22,24,32,33,36,46,50,53,58,70 \quad \mathrm{SH}$

$40 \quad \mathrm{SH}$

$33,61,65,70$

$1,4,8,12,17,20,21,22,24,32,33,36,44,45,49,53,58,64 \quad \mathrm{SH}$

$7 \quad$ SH

$3,4,7,16,26,31,33,37,41,42,44,45,47,55,56,57,61,65,67,70 \quad \mathrm{SH}, \mathrm{IH}$

$7 \quad$ SH

$3,4,7,9,12,19,26,48,51,54,64,65,68,70 \quad \mathrm{SH}, \mathrm{IH}$

$20,30,55$

1,44

30

44

67

12

$\mathrm{SH}$

$\mathrm{SH}$

$S$

$\mathrm{SH}$

$\mathrm{SH}$

$\mathrm{SH}$

$1,4,10,14,15,18,32,44,46,49,50,62$

$13,36,62,64 \quad \mathrm{SH}$

$7,8,10,14,16,21,25,26,36,46,47,64 \quad$ SH

$3,4,8,9,12,24,33,36,49,53,56,58,62,64 \quad \mathrm{SH}$

57

36,42

$12,36,43$

57

SH

$\mathrm{SH}$

$\mathrm{SH}$

$1,2,3,4,8,9,11,12,13,15,17,18,19,21,23,24,25,26$

$\mathrm{SH}, \mathrm{IH}$

$27,28,31,32,33,34,48$

$14,30,49,56$

16,30

$\mathrm{SH}, \mathrm{IH}$

$\mathrm{SH}, \mathrm{IH}$

$1,36,57$

$\mathrm{SH}, \mathrm{IH}$

$1,22,26,49,62 \quad \mathrm{SH}, \mathrm{IH}$

4

$\mathrm{SH}, \mathrm{IH}$

$11,30,55$

S

27,48

$\mathrm{AA}, \mathrm{SH}$

$3,4,8,12,18,20,21,22,26,27,28,30,31,32,33,36,37,40,41$. $43,44,46,48,50,52,53,55,57,58,62,64,67,69$

$1,3,4,15,17,18,20,21,24,27,31,32,33,36,40,48,61,63,64, \quad$ AA,SH

$3,17,18,19,23,26,27,32,41,42,47,51,55$

$\mathrm{SH}, \mathrm{IH}$

$2,11,14,17,20,28,31,42,51,54,59,62$

$\mathrm{IH}, \mathrm{M}$

$66, W$ coast of East Lewis I.

$7,11,14,17,28,38,42,51,59,62,66$

11

$\mathrm{IH}, \mathrm{M}$

$\mathrm{IH}, \mathrm{M}$

$1,3,13,15,18,21,22,24,26,27,32,40,43, \quad S H$ $44,48,50,52,53,57,60,67,69$

10

$18,26,27,45$

IH

$\mathrm{SH}$

$1,3,4,8,15,18,21,22,26,27,32,33,36,40,43,44,46,48,50$, $52,53,57,60,63,67,69$

$\mathrm{SH}$ 
Lima lima (Linnaeus, 1758)

Limaria basilanica (A. Adams and Reeve, 1850)

Limaria ?fragilis (Gmelin, 1791)

(WAM S 18945)

Limatula ?japonica (A. Adams, 1863)

(WAM S 18086)

\section{Subclass HETERODONTA}

Family Lucinidae

Anodontia edentula (Linnaeus, 1750)

Anodontia pila (Reeve, 1850)

Austriella corrugata (Deshayes, 1843)

Cavatidens omissa (Iredale, 1930)

Ctena bella (Conrad, 1834)

Divalucina cumingi (Adams and Angas, 1863)

Divaricella ornata (Reeve, 1850)

"Lucina" reevei Deshayes, 1863

Family Ungulinidae

Felaniella (Zemysia) sp. 1 (WAM S 18061)

Felaniella (Zemysia) sp. 2 (WAM S 18077)

?Felaniella sp. (WAM S 18059)

\section{Family Chamidae}

Chama fibula Reeve, 1846

Chama lazarus (Linnaeus, 1758)

Chama limbula Lamarck, 1819

Chama pacifica Broderip, 1834

Chama plinthota Cox, 1927

Chama pulchella Reeve, 1846

Pseudochama sp. (WAM S 18331)

Family Carditidae

Beguina semiorbiculata (Linnaeus, 1758)

Cardita incrassata Sowerby, 1825

Cardita ?crassicosta Lamarck, 1819 (WAM S 18101)

Cardita ?marmorea Reeve, 1843 (WAM S 18102)

Cardita murricata Sowerby, 1832

Cardita preissii Menke, 1843

Cardita ?variegata Bruguière, 1792 (WAM S 18104)

Venericardia cardiodes (Reeve, 1843)

"Venericardia"sp. (WAM S 12425)

Family Cardiidae

Acrosterigma angulata (Lamarck, 1819)

Acrosterigma dupuchense (Reeve, 1845)

Acrosterigma fultoni (Sowerby, 1916)

Acrosterigma reeveanum (Dunker, 1852)

Acrosterigma ?transcendens (Melvill and Standen, 1899) (WAM S 18199)

Acrosterigma wilsoni (Voskuil and Onverwagt, 1991)

Ctenocardia fornicata (Sowerby, 1840)

Fragum erugatum (Tate, 1889)

Fragum unedo (Linnaeus, 1758)

Fragum (Lunulicardia) retusum (Linnaeus, 1767)

Fulvia aperta (Bruguière, 1789)
$3,12,13,17,21,22,26,36,53,55,65$

$\mathrm{SH}$

$1,4,6,8,14,15,16,17,21,26,31,33,41,44,49,54,58$

$\mathrm{SH}$

DA1 Stn ?,53,58,67

SH

$33,36,38,67,70$

$\mathrm{SH}$
$11,14,35,54,62, W$ coast of East Lewis I.

$7,11,14,20,35$

31,54

$2,7,11,20$

$7,9,11,14,16,19,22,25,38,45,47,51,59,62,70$

$2,8,20$

$11,14,17$

$19,37,47$

$11,17,20,23,31,37,38,41,44,45,51,54,55,59,61,64,65$, $66,67,69, W$ coast of East Lewis I.

$14,31,56,70, W$ coast of East Lewis I.

$2,55,61$

$8,11,31,41,45,61,70$

12,32

$1,3,6,8,10,11,14,16,18,21,22,23,24,25,28,31,33,36,38$, $44,47,48,49,54,55,63,70$

$3,4,54$

$4,8,12,15,18,24,43,65,70$

23

$4,6,7,12,14,16,17,18,21,22,23,27,31,32,42,44,45,54$

$\mathrm{IH}, \mathrm{SH}$,

$\mathrm{SH}$

$\mathrm{IH}, \mathrm{SH}$

$\mathrm{SH}$

$\mathrm{IH}, \mathrm{SH}$

$\mathrm{SH}$

$\mathrm{IH}$

$1,3,4,12,17,21,22,24,26,31,36,44,47,48,49,61,62,63,64$

$\mathrm{SH}$

$7,30,56,65,67, W$ coast of East Lewis I.

$\mathrm{S}$

$20,25,28,31,48,70$

$\mathrm{SH}$

$7,11,14,20,23,25,28,31,38,45,51$

$1,4,6,7,10,12,15,16,17,21,24,31,33,36,37,40$

$42,45,47,54,63,65,70$

$10,23,30,56,59,62$

$1,30,31,48$

$\mathrm{SH}$

$20,28,30,55$

67

$\mathrm{S}$

$1,4,11,13,16,17,19,20,21,24,25,31,32,33$

$S$

$36,38,44,45,49,53,58,60,61,64$

$2,7,10,11,14,20,23,28,35,38,45,51,54,59,62,66,70 \quad S$

$20,23,28$

$7,9,10,11,16,17,30,31,33,37,38,42,44,45$,

$47,48,49,51,54,55,56,58,64,65,68,69,70$

$20,30,33$

$\mathrm{IH}$

$\mathrm{SH}, \mathrm{IH}$

$\mathrm{SH}$

$12,20,28,42,44,47,49,53,55,56,57,61$,

$62,64,65,67,68,69, \mathrm{~W}$ coast of East Lewis I.

$65,67,68$

$2,11,14,23,25,31,38,41,42,45,51,54,56,59,62,66,68,70$

$2,11,23,38,54,59,62$

$30,41,56,67,68$

$2,11,17,20,23,28,30,38,41,42,54,55,59,61,62,64$
S

S

S

S

$S$

S

S 
Fulvia sp. (WAM S 18205)

Laevicardium attenuatum (Sowerby, 1840)

Laevicardium biradiatum (Bruguière, 1789)

Nemocardium (Lyrocardium) lyratum

(Sowerby, 1840)

Plagiocardium setosum (Redfield, 1848)

Vepricardium ?multispinosum (Sowerby, 1838)

(WAM S 18246)

Family Hemidonacidae

Hemidonax arafurensis Ponder et al., 1981

Hemidonax donaciformis australiensis (Reeve, 184

$14,25,45, \mathrm{~W}$ coast of East Lewis I.

$\mathrm{S}$

S

Family Tridacnidae

Tridacna maxima (Röding, 1798)

Tridacna squamosa Lamarck, 1819

\section{Family Trapeziidae}

Trapezium bicarinatum (Schumacher, 1817)

Trapezium sowerbyi (Hidalgo, 1903)

Trapezium sublaevigatum (Lamarck, 1819)

Family Fimbriidae

Fimbria sowerbyi (Reeve, 1841)

14,25

$2,6,7,8,10,12,13,14,16,17,18,19,20,22,25,28,31,35,37$, $38,42,43,45,46,47,48,51,54,59,60,66$

$? 5,16,37,42,53,64$

$\mathrm{SH}, \mathrm{IH}$

$\mathrm{SH}, \mathrm{IH}$

$\begin{array}{ll}6,12,13,14,21,28,37,42,45,47,48,59,70 & \mathrm{SH} \\ 21 & \mathrm{SH} \\ 2,23,28 & \mathrm{SH}\end{array}$

\section{Family Mactridae}

Lutraria australis Reeve, 1854

Mactra (Electomactra) antecedens Iredale, 1930

Mactra (Mactra) cumingii Reeve, 1854

Mactra (Mactra) explanata Reeve, 1854

Mactra (Mactra) incarnata Reeve, 1854

Mactra (Mactra) luzonica Reeve, 1854

Meropesta nicobarica (Gmelin, 1791)

Spisula (Oxyperas) coppingeri (Smith, 1884)

Spisula (Oxyperas) triangularis (Lamarck, 1819)

Family Mesodesmatidae

Paphies (Atactodea) striata (Gmelin, 1791)

Family Donacidae

Donax faba Gmelin, 1791

$1,4,20,24,30,31,33,37,47,54,55,56,64,65,70$

S

$8,11,14,16,17,23,28,31,37,47,54,61,64,66$ 69

$2,7,9,11,14,31$

$57,65,67,69$

$7,8,9,14,16,37,48,51,70$

$2,7,11,20,23,30,31$

31,55

55

$\mathrm{S}$

$S$

S

$\mathrm{S}$

$S$

$\mathrm{S}$

$S$

$\mathrm{S}$

$25,28,38, W$ coast of East Lewis I.

S

$7,25,28,38,45, W$ coast of East Lewis I.

S

$23,36,38,42,51,59,62,67$

$20,31,55,65$

$31-S$

2,30

$11,20,23,28,41,69, \mathrm{~W}$ coast of East Lewis I. S

2

$2,23,54$

2

23,54

59

$2,11,31$

11

$11,20,30$

$17,37,41,49,61,64,67,69$

$7,10,11,14,18,19,20,23,25,28,30,35,37,38,41$,

$44,51,54,58,59,62,64,66,67$

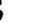

$S$

S

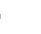

.

H,IH

$S$
$S$
$S$
$S$
$S$
$S$
$S$


Tellina (Tellinella) verrucosa Hanley, 1844 Tellina (Tellinella) virgata Linnaeus, 1758 Tellina (Tellinides) ovalis Sowerby, 1825

Tellina sp. (WAM S 18037)

Family Psammobiidae

Asaphis (Asaphis) violascens (Forskål, 1775)

Asaphis (Heteroglypta) contraria (Deshayes, 1843)

Gari (Dysmea) occidens (Gmelin, 1791)

Gari (Gari) anomala (Deshayes, 1858)

Gari (Gari) lessoni (Blainville, 1826)

Gari (Gari) maculosa (Lamarck, 1818)

Gari (Gari) sibogai Prashad, 1932

Gari (Gari) ?pallida (Deshayes, 1855)

(WAM S 18411)

Gari (Psammobia) amethysta (Wood, 1815)

Soletellina ?alba (Lamarck, 1818) (WAM S 30243)

Family Solecurtidae

Azorinus ?minutus (Dunker, 1861)

(WAM S 18935)

\section{Family Semelidae}

Leptomya psittacus Hanley, 1882

Semele jukesii (Reeve, 1853)

Semele ?casta A. Adams, 1853 (WAM S 18009)

Semele ?sinensis A. Adams, 1853 (WAM S 18275)

Semele exarata (A. Adams and Reeve, 1848)

Semele sp. (WAM S 18903)

\section{Family Solenidae}

Solen ?aureomaculatus Habe, 1964

(WAM S 18436)

Solen ?kajiyamai Habe, 1964 (WAM S 18434)

Solen ?roseomaculatus Pilsbry, 1901

(WAM S 18433)

Solen sp. 1 - see Lamprell and Healy 1998 \# 5232 (WAM S 18435)

Solen sp. 2 (WAM S 18439)

Family Pharidae

Cultellus attenuatus Dunker, 1861

Ensiculus cultellus (Linnaeus, 1758)

Family Veneridae

Anomalocardia squamosa (Linnaeus, 1758)

Antigona (Antigona) chemnitzii (Hanley, 1844)

Antigona (Antigona) lamellaris Schumacher, 1817

Antigona (Periglypta) resticulata Sowerby, 1853

Callista impar (Lamarck, 1818)

Callista (Costacallista) planatella (Lamarck, 1818)

Callista (Striacallista) ?phasianella

(Deshayes, 1854) (WAM S 18973)

Callista (Striacallista) ?roseotincta (Smith, 1885) (WAM S 30240)

Circe ?nana Melvill, 1898 (WAM S 30241)

Circe nummulina (Lamarck, 1818)

Circe scripta (Linnaeus, 1758)

Circe sulcata Gray, 1838

Circe tumefacta Sowerby, 1851

Clementia papyracea (Gray, 1825)

Dosinia altenai Fischer-Piette and Delmas, 1967
67

$2,11,23,38,51,54,59,62$

23

10

$\mathrm{S}$

$2,7,9,11,14,20,23,28,38,40$

S

$42,59,66,67, W$ coast of East Lewis I.

$43,49,55,62$

$1,4,10,12,13,15,20,23,31,33,41,42,44,47,49$,

$50,53,58,60,61,64,65,70$

$17,30,31,54,55,64$

$2,16,31$

$8,30,31,33,36,37,41,42,44,47,49,50,55,61,64,65$

$49,55,67,70$

13,23

$42,54,56,69$

W coast of East Lewis I.

11,20

$\mathrm{M}, \mathrm{S}$

$1,11,19,37,44,49,53,54,61,64,67$

$55, W$ coast of East Lewis I

$11,20,33,38,41,54,56,59,62$

$1,21,31,32,33,37,57,58,60$

$17,21,30,31.48,54,65,67,70$

S

$\mathrm{S}$

$S$

$S$

$S$

31,42

S

2,67

$\mathrm{S}$

$23,28,65, W$ coast of East Lewis I.

$\mathrm{S}$

2,11

S

17,67

S

67,69

$S$

$2,11,23,55$

$\mathrm{S}$

$2,11,23,31,54$

$\mathrm{S}$

$4,7,11,20,33,38,44,52,54,55,56,61,65$

$20,30,56,57,67$

$1,4,13,14,16,17,22,23,24,28,31,35,36,37,48,50$,

$52,53,55,58,63,64,65,67,70$

$2,23,38,51,54,62$

$S$

$S$

$S$

$S$

S

S

$S$

$S$

$12,54,56$

$2,11,23,38,51,54,61,67$

$41,55,56,61,67 \quad S$

59

$2,11,51,54,62$

$1,11,20,30,31,54,55,56,67 \quad S$

$41,51 \quad S$

62

$2,20,30,31,55,56$ 
Dosinia deshayesii A. Adams, 1855

Dosinia histrio Gmelin, 1791

Dosinia juvenilis (Gmelin, 1791)

Dosinia mira Smith, 1885

Dosinia ?sculpta (Hanley, 1845) (WAM S 18489)

Dosinia tumida (Gray, 1838)

Dosinia sp. (WAM S 18915)

Gafrarium menkei (Jonas, 1846)

Gafrarium tumidum Röding, 1798

Globivenus embrithes (Melvill and Standen,1899)

Globivenus toreuma (Gould, 1850)

Gomphina undulosa (Lamarck, 1819)

Irus ?irus (Linnaeus, 1758) (WAM S 18884)

Lioconcha ?annettae Lamprell and

Whitehead, 1990 (WAM S 22016)

Lioconcha fastigiata (Sowerby, 1851)

Marcia hiantina (Lamarck, 1819)

Paphia crassisulca (Lamarck, 1818)

Paphia semirugata (Philippi, 1847)

Paphia undulata (Born, 1780)

Paphia gallus (Gmelin, 1791)

Pitar affinis Gmelin, 1791)

Pitar (Pitarina) citrinus (Lamarck, 1818)

Pitar (Pitarina) pellucidus (Lamarck, 1819)

Placamen berryi (Gray, 1828)

Placamen gravescens (Menke, 1843)

Sunetta contempta Smith, 1891

Sunetta perexcavata Fulton, 1915

Tapes ?deshayesii (Hanley, 1844) (WAM S 30242)

Tapes dorsatus (Lamarck, 1818)

Tapes literatus (Linnaeus, 1758)

Tapes platyptycha Pilsbry, 1901

Tapes sericeus Matsukuma, 1986

Tapes sulcarius (Lamarck, 1818)

Tapes (Ruditapes) variegatus Sowerby, 1852

Tawera laticostata (Ohdner, 1917)

Family Petricolidae

Petricola ?divergens (Gmelin, 1791)

(WAM S 18726)

Petricola (Petricola) sp. (WAM S 18877)

Petricola (Velargilla) sp. (WAM S 30261)

Family Corbulidae

Corbula ?crassa Reeve, 1843 (WAM S 18927)

Corbula macgillivray (Smith, 1885)

Corbula ?tunicata Hinds, 1843 (WAM S 18924)

Family Gastrochaenidae

Cucurbitula cymbium (Spengler, 1783)

Gastrochaena (Gastrochaena) ?philippinensis

Deshayes, 1854 (WAMS 18728)

Gastrochaena ?tumidula Thiele, 1930 (WAM S 18729)

Gastrochaena (Spengleri) ?plicatilis Deshayes, 1854 (WAM S 30267)

Gastrochaena sp. (WAM S 30266)

Family Hiatellidae

Hiatella sp. (WAM S 30239)
65,69

$\mathrm{S}$

$20,23,31$

$30,37,43,44,49,54,55,56,58,61,63,65,67,69,70 \quad S$

$20,54,61,62, W$ coast of East Lewis I.

$11,17,23$

$28,31,42,55,56,61,62, \mathrm{~W}$ coast of East Lewis I.

$2,23,38,59$

$2,11,62$

$2,10,31,38,59$

$1,12,13,16,17,20,21,22,28,31,33,36,37,40,44,46,47,49$, $53,54,55,58,61,64,65,67,69,70$

$3,4,8,12,13,15,16,21,22,26,27,32,36,40,42,43,44$,

$46,47,50,52,53,55,58,67,70$

$7,9,12,14,25$

$1,6,11,15,17,38,48,59,64$

12

$12,20,23,24,28,30,31,38,40,41,42,44,54,55,56$,

$57,61,64,65,67,68,70$

2

$11,12,23,56$

$23,30,36,41,55,56,67,69, \mathrm{~W}$ coast of East Lewis I.

23,30

30,55

11

$2,11,23,59,62, \mathrm{~W}$ coast of East Lewis I.

59

51

$2,11,23,38,51,59, \mathrm{~W}$ coast of East Lewis I.

7

$42,57,67$

$2,8,16,38,44,67$

$2,11,17,20,23,25,30,31,33,61$

$1,2,7,11,38,55,62$

$3,4,7,11,13,15,16,27,31,32,33,36,44,55$

$1,2,4,10,11,16,17,19,23,24,25,28,31,33,37$,

$41,42,44,53,55,56,57,63,64$

$44,47,49,51,55,56,65,69,70$

$11,17,30,31,38,54,59,63,66, \mathrm{~W}$ coast of East Lewis I.

$13,14,17,25,33$

S

S

$\mathrm{IH}, \mathrm{S}$

$\mathrm{IH}, \mathrm{S}$

$\mathrm{S}$

S

$\mathrm{S}$ $\mathrm{SH}, \mathrm{IH}$ $\mathrm{S}$

S

$\mathrm{S}$

$\mathrm{S}$

S

$\mathrm{S}$

$\mathrm{S}$

$\mathrm{S}$

$\mathrm{S}$

$\mathrm{S}$

$S$

S

$\mathrm{S}$

$\mathrm{S}$

$\mathrm{S}$

S

$\mathrm{S}$

S

$\mathrm{S}$

S

$\mathrm{S}$

$\mathrm{S}$

$1,27,36,38,41,49$

$17,37,43,47,54,67 \quad \mathrm{SH}$

$42,54 \quad S$

$6,11,12,20,25,30,33,37,47,55,62,64, W$ coast of East Lewis I. S 16,56

$14,30,65,69$

$\mathrm{S}$

$\mathrm{S}$

36

$1,6,20,22,31,33,34,41,49,52,70 \quad \mathrm{SH}$

$1,4,11,31,42,52,63 \quad \mathrm{SH}$

$1,4,48,49$

$\mathrm{SH}$

1

$40,55,61$

$\mathrm{SH}, \mathrm{AA}$, 
Family Pholadidae

Parapholas ?quadrizonata (Spengler, 1792) (WAM S 18936)

Jouannetia cumingi (Sowerby, 1850)

$22,36,40,44,49,52,57,63,70$

$37,43,44$

Subclass ANOMALODESMATA

Family Thraciidae

Thracia imperfecta $($ Lamarck, 1818$)=$ alciope Angas, 1872

Family Laternulidae

Laternula ?valenciennesii (Reeve, 1860) (WAM S 18933)

Family Clavagellidae

Brechites vaginiferus australis (Chenu, 1843)

Family Myochamidae

Myadora ?complexa Iredale, 1924 (WAM S 18942) 23,54,55

Family Cleidothaeridae

Cleidothaerus sp. (WAM S 30271) (juvs)

Class SCAPHOPODA

Family Dentaliidae

Fissidentalium sp. (WAM S 30274) 
Appendix 2 Detailed descriptions of survey stations (DA1/98 and DA3/99) as applicable to the mollusc survey (for locality and other details see Station Lists).

\section{$\mathrm{DA1} / 98 / 01$}

Bottom of sand, dead shell and coral rubble, bottom depth averaged $6.0 \mathrm{~m}$, Porites bommies (largest $1.8 \mathrm{~m}$ high), smaller colonies of diverse corals, dead coral, algae; more algae and less coral towards beach, more sand over basement rock with sponges and less coral seaward.

\section{DA1/98/02}

Intertidal mud and muddy sand flats off sandy beaches and rocky points, mangles (Avicennia and Rhizophora) above rocks.

\section{DA1/98/03}

Bottom of silty sand, bottom depth averaged $9.0 \mathrm{~m}$, dissected edge of reef flat with reef top at $4.6 \mathrm{~m}$, rock boulders and coral bommies.

\section{DA1/98/04}

Bottom of silty sand, bottom depth averaged $11.0 \mathrm{~m}$, coral and rock bommies rising 1.0-3.0 $\mathrm{m}$ above bottom.

\section{DA1/98/05}

Intertidal reef (no specific mollusc sampling at this station).

\section{DA1/98/06}

Intertidal reef sampled at high water (water depth from $2.5-3.3 \mathrm{~m}$ ), rock platform with shallow gutters parallel to shore, silty sand and few flat rocks in gutters.

\section{DA1/98/07}

Intertidal sandy beach and muddy sand flat protected by offshore reef, exposed rocky point and boulders.

\section{DA1/98/08}

Intertidal reef at high tide with surface at $2.5 \mathrm{~m}$ and pools with silty sand to $5.5 \mathrm{~m}$, abrupt reef edge with spur and groove formation and sandy bottom between 7.7-9.0 m.

\section{DA1/98/09}

Sand over basement rock at $4.0 \mathrm{~m}$, abundant brown algae (Dictyota, Sargassum, etc.), slight depressions in rock containing sand, rubble and rocks, strong current of turbid water.

\section{DA1/98/10}

Intertidal beach rock and muddy gravel below mangal (Avicennia and Rhizophora) and above muddy sand flat with limestone rocks.

\section{DA1/98/11}

Intertidal muddy sand flat with mangal (mainly Avicennia, few Rhizophora) above, and increasing dead coral lumps below.

\section{DA1/98/12}

Bottom of silty sand, depth between 4.7-6.5 m; dead and living coral, coral slabs, short algal turf.

\section{DA1/98/13}

Rock pavement between $8.5-9.0 \mathrm{~m}$, dead and living coral with sand and shell rubble in pockets.

\section{DA1/98/14}

Intertidal sand flat, dead coral boulders with attached brown algae, fewer green and coralline algae.

\section{DA1/98/15}

Rock bottom from 7.9-19.3 m, steps of varied widths with vertical to undercut rises, luxuriant coral growth.

\section{DA1/98/16}

Flat bottom of sand rubble and rocks, bottom depth from $3.0 \mathrm{~m}$, little live coral, much brown algae.

\section{DA1/98/17}

Intertidal silty sand with shell and coral rubble and some rocks near rocky point, dead and living corals, soft corals, gorgonians, compound ascidians, etc.

\section{DA1/98/18}

Sandy bottom with coral bommies, bottom depth from 11.2 to approximately $13.0 \mathrm{~m}$.

\section{DA1/98/19}

Flat pavement rock with coral rock slabs, dead and living coral, algal turf with corallines and some green algae, turbid water from $2.0 \mathrm{~m}$ depth.

\section{DA1/98/20}

Intertidal flat of loose dead and living coral (mainly fungiids, with fewer small colonies of Pocillopora and faviids), sandy mud and little coral below low water spring tidal level, with very soft mud in places.

\section{DA1/98/21}

Coral bommies at $11.8 \mathrm{~m}$ with silty sand in hollows, mainly living corals.

\section{DA1/98/22}

Flat bottom with coral rubble and rocks at $4.9 \mathrm{~m}$ with Porites bommies, tabular and staghorn Acropora species.

\section{DA1/98/23}

Intertidal flat of very muddy sand, dead coral rocks increasing to landward, with fewer seaward, many sponges.

\section{DA1/98/24}

Flat bottom of silty sand with shell and coral rubble and living and dead coral slightly sloping from $4.9 \mathrm{~m}$, algal turf and coralline algae on rocks.

\section{DA1/98/25}

Exposed intertidal flat of coarse sand with large ripple marks, some shell and coral rubble; adjoining is a flat of dead coral rocks and silty sand.

\section{DA1/98/26}

Gradual stepped slope from $17.0-27.0 \mathrm{~m}$ of bare basalt encrusted with coralline algae, many soft corals, gorgonians, sponges and hard corals.

\section{DA1/98/27}

Flat pavement at $14.7 \mathrm{~m}$, with low live and dead coral colonies, gorgonians, hydroids and soft corals; turbid water.

\section{DA1/98/28}

Moderately steep intertidal shore of rocky points and sandy beach with mangal of Avicennia above and muddy sand to seaward.

\section{DA1/98/29}

Flat bottom at $4.0 \mathrm{~m}$ with live coral (staghorn and foliose Acropora and other groups), silty sand, some Porites bommies to $1.5 \mathrm{~m}$ high, and some brown algae.

\section{DA1/98/30}

Flat bottom at $11.0 \mathrm{~m}$ with very fine silty sand and shell grit, sponges and solitary ascidians, water very turbid. 


\section{DA1/98/31}

Intertidal flat of very silty sand and coral and igneous rocks, dense extensive mangal of Avicennia with deep mud.

\section{DA1/98/32}

Rock bottom at $11.7 \mathrm{~m}$ with little sand, dead and living corals, soft corals and algal turf.

\section{DA1/98/33}

Gently sloping basement rock from $7.2 \mathrm{~m}$ with silt and shell and coral rubble, with scattered hard and soft corals, sponges and gorgonians and algal turf.

\section{DA1/98/34}

Unofficial station (no mollusc survey).

\section{DA1/98/35}

Shallow area $(1.0 \mathrm{~m})$ live and dead coral and sand with shell and coral rubble.

\section{DA3/99/36}

Limestone pavement at $6.0-14.0 \mathrm{~m}$, with sand between low ridges, live branching, tabular and massive corals, some dead coral.

\section{DA3/99/37}

Sand, rubble and dead coral on pavement rock at $\sim 3.0$ $\mathrm{m}$, biota dominated by brown algae with some live tabular corals.

\section{DA3/99/38}

Intertidal and shallow subtidal sandy flat, with coral rubble, dead coral and rocks increasing shoreward; algal turf.

\section{DA3/99/39}

Hard bottom at $15.0-20.0 \mathrm{~m}$, large boulders with abundant cemented bivalves, few corals and other cnidarians, sandy patches between boulders.

\section{DA3/99/40}

Sloping rock reef at $\sim 6.0-9.0 \mathrm{~m}$, dissected at seaward edge with sandy floors to grooves at $\sim 14.0 \mathrm{~m}$, mainly soft and hard corals and cemented bivalves on rock walls of grooves.

\section{DA3/99/41}

Sloping sandy bottom from 1.0 to $\sim 4 \mathrm{~m}$ off shallow rock and live coral reef.

\section{DA3/99/42}

Intertidal survey of station DA3/99/41, with exposed rock reef with coarse sand, rubble and coral and rock boulders, intertidal sand surrounding reef.

\section{DA3/99/43}

Flat pavement rock from $\sim 12.0-16.0 \mathrm{~m}$, with number and size of boulders and coral bommies increasing with depth, cemented bivalves on sides of boulders.

\section{DA3/99/44}

Shallow rock bottom at $\sim 2.0 \mathrm{~m}$ with Porites bommies on sand and coral rubble bottom at $\sim 6.0 \mathrm{~m}$ depth, coral colonies increasing shoreward with cemented bivalves on vertical surfaces of rock or dead coral.

\section{DA3/99/45}

Intertidal sand flat with algal covered boulders, patches of sea grasses in deeper areas.

\section{DA3/99/46}

Limestone reef flat at $3.0 \mathrm{~m}$ edged with spur and groove formation, some sand, rubble and dead coral slabs in grooves at $\sim 10.0 \mathrm{~m}$, live corals mainly on vertical sides of grooves.

\section{DA3/99/47}

Sand overlying pavement rock at $\sim 5.0 \mathrm{~m}$, brown algae and coral colonies.

\section{DA3/99/48}

Wide intertidal rock pavement with dead coral and limestone rocks and shallow pools, abundant green and red algae, living and dead coral colonies

\section{DA3/99/49}

Rock and coral reef at $\sim .4 .0 \mathrm{~m}$, with sandy patches and abundant and diverse corals, sandy bottom becomes siltier seaward to $9.0 \mathrm{~m}$.

\section{DA3/99/50}

Rock bottom sloping from 16.0-20.0 m, seaward edge dissected and with corals and cemented bivalves on vertical faces, many large Porites bommies adjacent, giving way to sandy bottom sloping to $20.0 \mathrm{~m}$.

\section{DA3/99/51}

Wide intertidal muddy sand flats offshore from narrow mangal (Avicennia) and reef flat with shallow pools off rocky shore, algal dominated hard substrata.

\section{DA3/99/52}

Rock substrate from $12.0-20.0 \mathrm{~m}$, breaking up seaward and giving way to sandy bottom, abundant hard and soft corals, cemented bivalves and other attached forms on rock, few molluscs in soft substratum.

\section{$\mathrm{DA} 3 / 99 / 53$}

Rock substrate at $5.0 \mathrm{~m}$ covered with sand and rubble, with slope to $\sim 7.0 \mathrm{~m}$. Large Porites bommies, some other hard corals, much soft coral and gorgonians, water very turbid, algae increasing inshore.

\section{DA3/99/54}

Wide intertidal sandy mud flat off rocky shore and mangal at $S$ end of large bay, abundant and diverse molluscs on and in soft substratum.

\section{DA3/99/55}

Bottom of coarse sand covering flat pavement rock at $17.0 \mathrm{~m}$, "sponge garden" with many gorgonians and some hard and soft corals, abundant infaunal bivalves, strong current of very turbid water.

\section{DA3/99/56}

Undulating pavement rock at $\sim 10.0 \mathrm{~m}$, with silty sand and rubble between very low ridges, many gorgonians and sponges on ridges, molluscs abundant and diverse, strong current of turbid water.

\section{DA3/99/57}

High energy area of rock substratum at $\sim 8.0-13.0 \mathrm{~m}$, deep crevices floored with mobile coarse sand, hard and soft corals and cemented bivalves on vertical rock faces, some sand-dwelling bivalves.

\section{DA3/99/58}

Shallow subtidal sand with some rubble at $\sim 3.0 \mathrm{~m}$, with rock and coral reef, diverse hard corals, moderate diversity of hard and soft substratum molluscs.

\section{DA3/99/59}

Intertidal sand and rubble flat seaward of narrow mangal, sand more silty towards mangal, less so near rocky points, high diversity and abundance of hard and soft substratum molluscs. 


\section{DA3/99/60}

Bay with shallow rock and coral reef sheltering sandy lagoon, diverse and abundant hard corals, particularly Pavona, abundant molluscs (with limited diversity) particularly in and around Pavona.

\section{DA3/99/61}

Flat bottom at $\sim 4.5 \mathrm{~m}$ covered with sand, rubble and some dead coral slabs, much brown algae particularly Dictyota, with little coral except for area of low Porites colonies, molluscs relatively diverse but not abundant, water turbid.

\section{DA3/99/62}

Intertidal muddy sand flat in bay with rocky points, landward of the flat is a fairly deep mangal of Avicennia, Rhizophora and Bruguiera. Abundant and fairly diverse molluscs.

\section{DA3/99/63}

Rock and coral reef from $\sim 2.0-5.0 \mathrm{~m}$, with diverse hard corals including Porites bommies in deeper areas, shallow rocks with algal turf.

\section{DA3/99/64}

Subtidal sand over pavement rock at $\sim 5.0 \mathrm{~m}$, with hard and soft corals; more rocks and algae inshore backed by deep mangal, abundant and diverse molluscs.

\section{DA3/99/65}

Undulating pavement rock at $\sim 13.0-15.0 \mathrm{~m}$ with sand and rubble between low ridges, a "sponge garden" with many gorgonians and some small colonies of hard and soft corals, mollusc diversity high, infaunal species abundant.

\section{DA3/99/66}

Intertidal mangal with tidal creek, relatively low abundance and diversity of molluscs and other groups.

\section{DA3/9967}

Sloping pavement rock at $\sim 17.5-20.0 \mathrm{~m}$, with little rubble between low ridges, low-growing sponges and gorgonians with some large Porites bommies and small colonies of other hard corals, some species of cemented and of infaunal bivalves abundant.

\section{DA3/99/68}

Subtidal sand plain at $6.5 \mathrm{~m}$, relatively high diversity of infaunal bivalves, high abundance of echinoids, some green and brown algae.

\section{DA3/99/69}

Rock boulders covering gas pipeline rising to $15.0 \mathrm{~m}$ above sand plain at $\sim 18.0 \mathrm{~m}$, relatively few small colonies of hard corals, abundant cemented bivalves, diverse bivalves on and in sand substratum.

\section{DA3/99/70}

Limestone pavement at $\sim 6.0 \mathrm{~m}$ with covering of sand and rubble, some algae and small colonies of hard corals, moderate diversity and abundance of cemented and infaunal mollusc species. 